\title{
STRUCTURE OF SPECTRAL MEASURES ON LOCALLY CONVEX SPACES $\left({ }^{1}\right)$
}

\author{
BY \\ BERTRAM WALSH
}

0. Introduction. Though spectral measures - i.e. countably additive idempotent-valued set functions defined on a $\sigma$-algebra of sets with values in $\mathscr{L}(E)-$ form the nominal topic of this paper, it can be looked on as having for its twin objects of study the spectral operators and the Boolean algebras of projections on locally convex spaces, since both are intimately connected with spectral measures - the operators with their "resolutions of the identity," the Boolean algebras (if "complete" in a suitable sense) with the measures, defined on the Stone spaces of the algebras, whose values are the projections making up the algebras. Such operators and algebras, acting on Banach spaces, have received a great deal of attention since the operators were introduced and exhaustively investigated in the six papers [1], [2], [7], [11], [19] and [20] and the same service performed for the Boolean algebras in [3] and [4]. The concepts of spectral operator and Boolean algebra of projections are not (at least a priori) connected with normability of the topology of the vector space on which the operator or algebra acts; the definitions usually given for these objects acting on Banach spaces can be extended to general locally convex spaces in a perfectly straightforward way, thus yielding the objects of study of this paper. Presumably the Banach-space techniques which establish the fundamental properties of the associated spectral measures could be imitated and the corresponding fundamental properties of these operators and algebras deduced. In order to derive our principal results, however, we have been obliged to investigate these concepts from a geometrical, topological and order-theoretic point of view, thus continuing the line of research begun in [17]. Aside from our principal results, a consequence of adopting this point of view is a fairly immediate determination of the limits to which many rather deep theorems concerning spectral measures acting on Banach spaces can be generalized for measures acting on locally convex spaces, coupled with immediate and natural proofs of the generalizations and thus a simplification of technique even for Banach spaces. The main theorems of $\S \S 2$ and 4 , however, represent indications of the restrictedness rather than the generality of the concept of spectral measure, and thus the restrictedness

Received by the editors December 22, 1964.

(1) Part of the material of this paper formed part of the author's 1963 University of Michigan thesis, written under the direction of H. H. Schaefer while the author held an NSF Coöperative Fellowship. The author wishes to thank Professor Schaefer for his advice and encouragement. The other research reported, and the preparation of the paper, were carried out under NSF Grant GP-4563. 
of the classes of spectral operators and Boolean algebras of idempotents: we establish that under fairly general circumstances a spectral measure acting on a locally convex space $E$ can be viewed as a projective limit of spectral measures acting on a family of Banach spaces whose projective limit is (the completion of) $E$, and thus that spectral operators and Boolean algebras of projections are intrinsically connected with normability, and we show that in case $E$ is a nuclear or Montel space, such a spectral measure (or a natural extension of it) must be purely atomic. Thus we show, roughly, that for these spaces there is no more general spectral operator than a sum of multiples of mutually orthogonal projections, and no more general Boolean algebra of complemented subspaces than a purely atomic one.

$\$ 1$ lists a number of important basic definitions and theorems (with a few proofs of the latter) and establishes conventions of notation and language. Some supplementary results on nuclear spaces are given; these are restatements, in a form suitable for use in the present setting, of theorems of Grothendieck [9]. General information on locally convex spaces which we will use without explicit reference is to be found in [6] and [12].

A useful property which spectral measures, or more generally $\sigma$-complete Boolean algebras of commuting idempotents, must possess under reasonable conditions (tonnelé, metrizable) on the spaces on which they act is that their values (or the idempotents themselves) form an equicontinuous family of operators; $\$ 2$ investigates some consequences of this property. Propositions 2.1 and 2.3 establish a property of the space as a module over the algebra of bounded measurable functions which is analogous to local convexity over the scalars; 2.5 establishes a similar, stronger result for an important special class of locally convex spaces. A few applications of these results are made. In $\S 3$ we show that the cyclic subspaces of a spectral measure carry an order structure induced in a natural way by the measure, and that this order has very strong and useful properties; in fact, there is a far-reaching analogy between cyclic subspaces and $L^{1}$-spaces of totally finite measures, which is very close and concrete for metrizable spaces and surprisingly strong even in the nonmetrizable case. For Fréchet spaces and their strict inductive limits this is connected with a representation of the cyclic subspaces as spaces of classes of measurable functions; this representation does not appear to have been described before, although some of the consequences of its existence have been investigated and are in fact basic to the study of multiplicity of spectral measures on Banach spaces, as in [4]. Using the order structure one can place these and other results of Bade in a general context, determine the limits of possible generalization, and obtain natural proofs of the generalizations, as in Corollary 3.10 and the following material. In $\S 4$, finally, this representation and order structure enable us to show that a Borel spectral measure on a Montel space must be purely atomic. Using this highly restrictive structural result, we construct a rather concrete representation for 
the cyclic subspaces of a spectral measure on a nuclear space and completely characterize the spectral operators and measures on $\omega_{d}$ and $\phi_{d}$, the product and locally convex direct sum of $d$ copies of the complex numbers respectively.

1. Preliminaries. $\boldsymbol{R}$ denotes the real numbers, $\boldsymbol{C}$ the complex numbers, $C^{*}$ the Riemann sphere. A locally convex space in addition to being what its name implies is assumed Hausdorff unless the contrary is explicitly stated. If $E$ is locally convex and $p$ is a continuous seminorm on $E$, the space $E / p^{-1}[0]$ with the topology generated by the norm induced on it by $p$ will be denoted by $E_{p}$ and its completion (like all completions) by $E_{p}{ }^{\wedge}$; the image of an element $x \in E$ under the natural mapping from $E$ to $E_{p}{ }^{\wedge}$ will be denoted by $x_{p}$. Similarly, if $S$ is a bounded convex circled subset of $E$, then $E_{S}$ will denote the vector subspace (not necessarily closed) of $E$ generated by $S$, under the norm topology given by the gauge (Minkowski functional) of $S$ on $E_{S}$. We note that if $E$ is sequentially complete and $S$ is closed, then $E_{S}$ is a Banach space. We use the following abbreviations: an $\mathscr{F}$-space is a Fréchet space (complete metrizable locally convex), and an $\mathscr{L} \mathscr{F}$-space a space which can be represented as the topological inductive limit of an increasing sequence of $\mathscr{F}$-spaces; an $s \mathscr{L} \mathscr{F}$-space is a strict topological inductive limit of $\mathscr{F}$-spaces. We recall that a Montel space is a tonnelé space each of whose bounded subsets is relatively compact (in the "strong" topology of the space); such spaces are necessarily reflexive. The topology of pointwise convergence, or simple convergence, on $\mathscr{L}(E)$ is referred to as the strong operator topology, and the corresponding weak topology, which is the one induced by the duality between $\mathscr{L}(E)$ and $E \otimes E^{\prime}$, is referred to as the weak operator topology. The phrase weakly sequentially complete, when predicated of a locally convex space $E$, means that every sequence in $E$ which is weakly $\left(=\sigma\left(E, E^{\prime}\right)\right)$ Cauchy is weakly convergent to some element of $E$.

Ordered (real) locally convex spaces are our principal measure-theoretic investigative tool. It is clear that the convex cone (with vertex zero) of positive elements of such a space determines the order, so one can describe properties of the order in terms of properties of the cone. If $E$ is an ordered locally convex space with topology $\mathfrak{I}$ and positive cone $K$, one says that $K$ is normal with respect to $\mathfrak{I}$ if there exists a family of continuous seminorms generating $\mathfrak{I}$ which are monotone on $K$. Normality can be characterized dually by considering the cone $K^{\prime} \subseteq E^{\prime}$ consisting of the continuous linear forms which are nonnegative on $K: K$ is normal if and only if each equicontinuous subset $S \subseteq E^{\prime}$ is contained in $T-T$ for some equicontinuous subset $T \subseteq \mathrm{K}^{\prime}$. Thus $K$ normal implies $E^{\prime}=K^{\prime}-K^{\prime}$ and that, in turn, is equivalent to normality of $K$ for $\sigma\left(E, E^{\prime}\right)$. It is easy to see that if a cone is weakly $\left(=\sigma\left(E, E^{\prime}\right)\right)$ normal, then order intervals in the corresponding order are bounded subsets of $E$, and that whenever $\left\{x_{\alpha}\right\},\left\{y_{\alpha}\right\}$ and $\left\{z_{\alpha}\right\}$ are generalized sequences (with the same index set) for which $x_{\alpha} \leqq y_{\alpha} \leqq z_{\alpha}$ for all $\alpha$ and $x_{\alpha} \rightarrow x, z_{\alpha} \rightarrow x$ in a topology $\mathfrak{I}$ for which 
the cone is normal, then $y_{\alpha} \rightarrow x$ in $\mathfrak{I}$ also. An important property of normal eones is given by the

Monotone Convergence Theorem 1.1 [Dini-Schaefer]. Let $K$ be normal in $E[\mathfrak{T}]$, and let $M \subseteq E$ be directed under the order $\leqq$ corresponding to $K$. If the filter of "ends" of $M$ converges to $x_{0} \in E$ weakly, then it also converges to $x_{0}$ in the topology $\mathfrak{I}[15]$.

If $u$ is a linear transformation defined on a dense subspace $D_{u}$ in a locally convex space $E$ with values in $E$, we define the resolvent set of $u$ to be the set of all complex numbers $\lambda$ possessing neighborhoods $N_{\lambda}$ such that for each $\mu \in N_{\lambda}$, the linear transformation $(\mu \cdot 1-u)$ is $1-1$ and has range dense in $E,(\mu \cdot 1-u)^{-1}$ has a (necessarily unique) extension $R(\mu) \in \mathscr{L}(E)$ and the function $\mu \rightarrow R(\mu)$ is analytic in a neighborhood of $\lambda$, namely $N_{\lambda}$; if $R(\cdot)$ is analytic in a neighborhood of $\infty$ then we adjoin $\infty$ to the resolvent set, since it easily follows that $R(\cdot)$ cannot have an isolated singularity at $\infty$ if $u$ is densely defined. We denote the resolvent set of $u$ by $\rho(u)$ and its complement by $\sigma(u)$, calling the latter the spectrum of $u$. If the resolvent set is nonempty, then the resolvent satisfies the resolvent equation $R(\lambda)-R(\mu)=-(\lambda-\mu) R(\lambda) \cdot R(\mu)$ for $\lambda$ and $\mu$ in $\rho(u)$, and using this fact one can construct (in the presence of very mild completeness properties of $E$ ) an operational calculus for functions (locally) analytic in neighborhoods of $\sigma(u)$, i.e. a homomorphism of the algebra of functions (locally) analytic in neighborhoods of $\sigma(u)$ into $\mathscr{L}(E)$. The relations between this operational calculus and the operational calculus for continuous functions on $\sigma(u)$ when $u$ is a spectral operator on locally convex $E$ are discussed in [17]. The point spectrum of $u$ is the set of complex numbers $\lambda$ for which $\lambda-u$ is not $1-1$, and it is denoted hereinafter by $\pi(u)$.

The concept "Boolean algebra of commuting idempotents in $\mathscr{L}(E)$ "' is just as meaningful for locally convex spaces as it is for Banach spaces; the union and intersection operations are defined via the algebraic operations in $\mathscr{L}(E)$, viz. $e \vee f=e+f-e f$ and $e \wedge f=e f$, and correspond to range-inclusion. The notions of abstract $\sigma$-completeness, $\sigma$-completeness and completeness used by Bade in [3] are topological and algebraic, and consequently extend themselves immediately to the locally-convex-space setting. Instead of considering bounded Boolean algebras of projections on Banach spaces, we consider equicontinuous Boolean algebras on locally convex spaces. For metrizable locally convex spaces, one can easily imitate the proof of Theorem 2.2 of [3] and show that

Proposition 1.2. If $E$ is a metrizable locally convex space, $\mathfrak{A}$ a Boolean algebra of projections in $\mathscr{L}(E)$ which is $\sigma$-complete as an abstract Boolean algebra, then $\mathfrak{U}$ is equicontinuous.

Proof Sketch. It suffices to show that for any bounded circled convex subset $B \subseteq E$ the set $\bigcup_{e \in \mathfrak{A}} e[B]$ is bounded. If the contrary were true for some such $B$ 
then there would exist a continuous seminorm $p$ on $E$ for which $\sup \{p(e x) \mid e \in \mathfrak{A}, x \in B\}=\infty$, and as in [3] there would exist in consequence a collection of mutually disjoint sequences $\left\{h_{j k}\right\}_{k \in N}$ of projections in $\mathfrak{A}, j=1,2, \cdots$, with $\lim _{k}\left(\sup \left\{p\left(h_{j k} x\right) \mid x \in B\right\}\right)=\infty$ for all $j \in N$. Set $f_{j}=\bigvee_{k \in N} h_{j k}$; then since $h_{j k}=h_{j k} f_{j}$, it is also true that $\lim _{k}\left(\sup \left\{p\left(h_{j k} x\right) \mid x \in f_{j}[B]\right\}\right)=\infty$. Moreover, since each set $f_{j}[B]$ is circled and bounded, for any (fixed) neighborhood base $\left\{U_{j}\right\}_{j \in N}$ of zero it is true that $\lim _{k}\left(\sup \left\{p\left(h_{j k} x\right) \mid x \in f_{j}[B] \cap U_{j}\right\}\right)=\infty$. Selecting for each $j \in N$ an $x_{j} \in f_{j}[B] \cap U_{j}$ and an $h_{j, n_{j}}$ with $p\left(h_{j, n_{j}} x_{j}\right)>j$, then setting $f=\bigvee_{j \in N} h_{j, n_{j}}$, we have $p\left(f x_{j}\right)=p\left(f f_{j} x_{j}\right)=p\left(h_{j, n_{j}} x_{j}\right)>j$, so $f \in \mathfrak{A}$ sends a null sequence into a non-null sequence and thus fails to be continuous, a contradiction.

A slight modification of the same proof gives the same conclusion for an abstractly $\sigma$-complete Boolean algebra $\mathfrak{A}$ of projections on an $s \mathscr{L} \mathscr{F}$-space for which each of the sets $\{e x \mid e \in \mathfrak{A}\}, x \in E$, is contained in one of the limitands in the inductive limit making up the space $E$.

The same simple approximation argument used in the Banach-space case shows

Proposition 1.3. If $\mathfrak{A}$ is a complete equicontinuous Boolean algebra of projections on a locally convex space $E,\left\{e_{\alpha}\right\} \subseteq \mathfrak{A}$ a family of elements of $\mathfrak{A}$ directed (upward or downward respectively) in the natural ordering of $\mathfrak{A}$, then $\lim _{\alpha} e_{\alpha}=\bigvee_{\alpha} e_{\alpha}$ or $\lim _{\alpha} e_{\alpha}=\bigwedge_{\alpha} e_{\alpha}$ respectively in the strong operator topology, and similarly for $\mathfrak{A} \sigma$-complete and $\left\{e_{\alpha}\right\}$ countable.

Just as in the Banach-space situation, a $\sigma$-complete or complete Boolean algebra of projections satisfying the conclusion of this proposition - in particular an equicontinuous one - may be regarded as an $\mathscr{L}(E)$-valued measure, defined on the Baire or Borel sets respectively of its Stone space and countably additive in the strong operator topology.

Let $E[\mathfrak{T}]$ be a locally convex space, $X$ a set, $\mathfrak{S}$ a $\sigma$-algebra of subsets of $X$.

Definition 1.4. A spectral measure triple in $\mathscr{L}(E), E$ locally convex, is a triple $(X, \mathfrak{S}, \mu)$ where $\mu$ is a set function from $\mathfrak{S}$ to $\mathscr{L}(E)$ which is countably additive in the weak operator topology and satisfies:

(1) $\mu(X)=1 \in \mathscr{L}(E)$;

(2) for $\delta_{1}$ and $\delta_{2}$ in $\mathfrak{S}, \mu\left(\delta_{1} \cap \delta_{2}\right)=\mu\left(\delta_{1}\right) \cdot \mu\left(\delta_{2}\right)$.

We use these abstract set functions to define a spectral measure rather than following the approach of [17] and defining such a measure to be a weakly continuous homomorphism from the uniform-norm algebra of continuous functions on a compact Hausdorff space into $\mathscr{L}(E)$, because the weak-sequentialcompleteness hypotheses of [17] can be avoided if a set function and countable additivity are already present, as they are for example when the spectral measure arises from a $\sigma$-complete Boolean algebra of commuting idempotents in $\mathscr{L}(E)$. 
We observe that such a measure must take its values in a weakly normal cone in $\mathscr{L}(E)$, namely the convex cone generated by its values: if $x \in E$ and $x^{\prime}$ is a real-linear continuous form on $E$, then the set function $\delta \rightarrow\left\langle\mu(\delta) x, x^{\prime}\right\rangle$ is a finite-valued signed measure on $\mathfrak{S}$ and hence there is a set $\varepsilon \in \mathbb{S}$ for which $\delta \rightarrow\left\langle\mu(\delta \cap \varepsilon) x, x^{\prime}\right\rangle$ is a nonnegative finite-valued measure and $\delta \rightarrow\left\langle\mu(\delta \sim \varepsilon) x, x^{\prime}\right\rangle$ a nonpositive finite-valued measure (Hahn decomposition). Thus for every $\delta \in \mathbb{S}$ (the ' denoting transposition, as usual)

$$
\left\langle\mu(\delta) x, x^{\prime}\right\rangle=\left\langle\mu(\delta) x, \mu(\varepsilon)^{\prime} x^{\prime}\right\rangle+\left\langle\mu(\delta) x, \mu(X \sim \varepsilon)^{\prime} x^{\prime}\right\rangle
$$

and so $x \otimes x^{\prime} \in \mathscr{L}(E)^{\prime}$ can be decomposed into the difference of two weakly continuous linear forms on $\mathscr{L}(E)$ which are positive with respect to the cone previously described. It follows readily that the values of $\mu$ are bounded in the strong operator topology; if $E$ is tonnelé, then, they form an equicontinuous subset of $\mathscr{L}(E)$, so that in this case Proposition 1.3 has a converse - the property $\lim _{n} e_{n}=\bigvee_{n=1}^{\infty} e_{n}$ for increasing sequences $\left\{e_{n}\right\}$ in $\mathfrak{A}$ implies its equicontinuity. At any rate, if the values of a spectral measure do form an equicontinuous subset of $\mathscr{L}(E)$, then the decomposition above carries equicontinuous sets of elements $x^{\prime}$ to equicontinuous sets of elements $\mu(\varepsilon)^{\prime} x^{\prime}$ and $\mu(X \sim \varepsilon)^{\prime} x^{\prime}$ (though $\varepsilon$ depends on $x^{\prime}$ ) so that the cone generated by the $\mu(\delta), \delta \in \mathbb{S}$, is normal in the strong operator topology. It follows easily that under these circumstances $\mu$ must be countably additive in the strong operator topology: for the partial sums of the elements of a sequence of values of $\mu$ on disjoint sets in $\subseteq$ converge weakly monotonically to the measure of the union, thus strongly by Theorem 1.1.

Say that the triple $(X, \mathfrak{S}, \mu)$ is equicontinuous if the values of $\mu$ on $\subseteq$ are equicontinuous. Given such an equicontinuous triple in $\mathscr{L}(E)$ with $E$ sequentially complete in its topology, there is no problem in defining the integrals of bounded S-measurable functions on $X$ : what the integrals of simple functions should be is clear, and if $f$ is a real-valued simple function with $-\lambda \leqq f(\cdot) \leqq \lambda$ on $X$, then also $-\lambda \cdot 1 \leqq \int f d \mu \leqq \lambda \cdot 1$ in the order induced by $\mu$ on $\mathscr{L}(E)$; if both ends of this inequality converge to zero in the strong operator topology, then by normality so does $\int f d \mu$, showing that $f \rightarrow \int f d \mu$ is continuous in the uniform norm on $X$ to $\mathscr{L}(E)$, whence it may be extended to the bounded $\subseteq$-measurable functions by density, continuity, and the sequential completeness of $E$. The extension to complex-valued bounded $\mathfrak{S}$-measurable functions is immediate. The integral in addition to being linear is multiplicative, since for simple $f$ and $g$ the relation $\int f g d \mu=\int f d \mu \cdot \int g d \mu$ is an immediate consequence of the condition (2) on spectral measures. The integral thus represents the bounded $\mathfrak{S}$-measurable $\boldsymbol{C}$-valued functions as an algebra of operators on $E$, which algebra we shall call the spectral algebra associated with $(X, \subseteq, \mu)$. Clearly each element of such an algebra is a spectral operator in the sense of [17], and by a change of measure (setting $v=\mu \circ f^{-1}$ ) we can write any operator $t=\int f d \mu$ as $\int z d v$, where $z$ 
is the identity function on $C$; it is known that the measure $v$ depends only on the operator $t$ [17] and that its support (a compact subset of $C$ ) is the spectrum of $t$.

In addition to the spectral algebra associated with $(X, \mathfrak{S}, \mu)$ we consider other spectral operators related to it: these are the unbounded spectral operators associated with $(X, \mathfrak{S}, \mu)$. Defining these operators requires a notion of integrability of $\subseteq$-measurable functions $f$ with respect to the $E$-valued measures $\delta \rightarrow \mu(\delta) x$, where $x \in E$, namely the following: say that $f$ is weakly integrable if for each $x^{\prime} \in E^{\prime}, f$ is integrable with respect to the (not necessarily nonnegative scalar-valued measure $\delta \rightarrow\left\langle\mu(\delta) x, x^{\prime}\right\rangle$ and the linear form $x^{\prime} \rightarrow \int f d\left\langle\mu(\cdot) x, x^{\prime}\right\rangle$ is $\sigma\left(E^{\prime}, E\right)$-continuous, or equivalently if there exists $y \in E$ for which $\langle y, x\rangle=\int f d\left\langle\mu(\cdot) x, x^{\prime}\right\rangle$ for all $x^{\prime} \in E^{\prime}$; denote that $y$ by $\int f d \mu(x)$ and call it the weak integral of $f$. Now, given an $\mathfrak{S}$-measurable $\boldsymbol{C}$-valued function $f$ defined on $x$, we can define a linear transformation by $x \rightarrow \int f d \mu(x)$ on that linear subspace $D_{f}$ of $E$ for which $f$ is weakly integrable with respect to $\mu(\cdot) x$; it can be shown that $D_{f}$ is dense in $E$ and that the linear transformation so defined is closed [17]. The change of measure $v=\mu \circ f^{-1}$ again represents the operator as $x \rightarrow \int z d v(x)$; once again the support of $v$ on $C^{*}$ is the spectrum of this operator, and the operator determines $v$ uniquely if the resolvent set of the operator is nonempty.

Two other objects closely related to spectral measure triples will occur in what follows: the pseudo-unitary group associated with $(X, \Im, \mu)$ is the subset $\left\{\int f d \mu \mid f\right.$ S-measurable, $\left.|f| \equiv 1\right\}$ of $\mathscr{L}(E)$; it is evidently an abelian group under composition. The cyclic subspace generated by $x \in E$ is the smallest closed subspace of $E$ containing $\{\mu(\delta) x \mid \delta \in \mathbb{S}\}$, the real cyclic subspace generated by

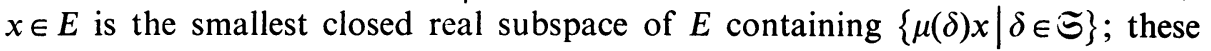
are denoted by $\mathfrak{M}(x)$ and $\mathfrak{M}_{R}(x)$ respectively.

A linear transformation $u: E \rightarrow F$ between locally convex spaces is nuclear if there exist an equicontinuous subset $\left\{x^{\prime}{ }_{i}\right\}_{i \in N}$ of $E^{\prime}$, a subset $\left\{y_{i}\right\}_{i \in N}$ of a bounded circled convex subset $S$ of $F$ for which $F_{S}$ is complete, and a sequence $\left\{\lambda_{i}\right\}_{i \in N} \in l^{1}$ for which $u x=\sum_{i \in N} \lambda_{i}\left\langle x, x_{i}^{\prime}\right\rangle y_{i}$ for every $x \in E$. Clearly the $\left\{\lambda_{i}\right\}_{i \in N}$ may be chosen positive. A locally convex space is nuclear if for each continuous seminorm $p$ the natural mapping $E \rightarrow E_{p}{ }^{\widehat{ }}$ is nuclear. It follows that a locally convex space is nuclear if and only if to each continuous seminorm $p$ on $E$ there corresponds at least one seminorm $q$ and positive $k \in \boldsymbol{R}$ for which $p(x) \leqq k \cdot q(x)$ for all $x \in E$ such that the natural mapping $E_{q} \widehat{ } \rightarrow E_{p} \widehat{ }$ is nuclear. The following proposition is implicit in [9]:

Proposition 1.5. For each nuclear space $E$ there exists for each extended real number $p, 1 \leqq p \leqq \infty$, a family of linear mappings of $E$ into $l^{p}$ with the property that the topology of $E$ is the coarsest topology for which all mappings of the family are continuous.

Proof. For each continuous seminorm $r$ on the nuclear space $E$ we shall 
exhibit a continuous linear mapping $t: E \rightarrow l^{p}$ and constant $m$ for which $r(x) \leqq m \cdot\|t x\|_{p},\|\|_{p}$ denoting the usual norm on $l^{p}$. Since $E$ is nuclear there exists a continuous seminorm $r_{1}$ on $E$ and positive constant $k$ for which $r(x) \leqq k \cdot r_{1}(x)$ for $x \in E$ such that the natural mapping $E_{r_{1}}{ }^{\wedge} \rightarrow E_{r}{ }^{\wedge}$ is nuclear: let the natural mapping be given by

$$
x \rightarrow \sum_{i \in N} \lambda_{i}\left\langle x, x_{i}{ }^{\prime}\right\rangle y_{i}
$$

with $\left\{x_{i}^{\prime}\right\}_{i \in N}$ equicontinuous in $\left(E_{r_{1}}{ }^{\wedge}\right)^{\prime},\left\{y_{i}\right\}_{i \in N}$ bounded in $E_{r}{ }^{\wedge}$, each $\lambda_{i}>0$ and $\sum_{i \in N} \lambda_{i}<\infty$. The mapping $u: E_{r_{1}}{ }^{\wedge} \rightarrow l^{p}$ defined by

$$
u x=\left(\left(\lambda_{1}\right)^{1 / p}\left\langle x, x_{1}^{\prime}\right\rangle,\left(\lambda_{2}\right)^{1 / p}\left\langle x, x_{2}^{\prime}\right\rangle, \cdots\right)
$$

(with the convention that $\lambda^{1 / p}=1$ for $p=\infty$ ) is easily seen to be continuous by the equicontinuity of the $\left\{x_{i}^{\prime}\right\}_{i \in N}$. On the other hand (with $q$ denoting, as usual, the solution of $1 / p+1 / q=1$ )

$$
\begin{aligned}
r\left(\sum_{i \in N} \lambda_{i}\left\langle x, x_{i}^{\prime}\right\rangle y_{i}\right) & \leqq \sum_{i \in N} \lambda_{i}\left|\left\langle x, x_{i}^{\prime}\right\rangle\right| \cdot r\left(y_{i}\right) \\
& =\sum_{i \in N}\left(\lambda_{i}\right)^{1 / p}\left|\left\langle x, x_{i}^{\prime}\right\rangle\right| \cdot\left(\lambda_{i}\right)^{1 / q} \cdot r\left(y_{i}\right) \\
& \leqq\|u x\|_{p} \cdot\left[\sum_{i \in N} \lambda_{i}\right]^{1 / q} \cdot \sup _{i} r\left(y_{i}\right)
\end{aligned}
$$

and thus, taking $m=\left[\sum_{i \in N} \lambda_{l}\right]^{1 / q} \cdot \sup _{i} r\left(y_{\imath}\right)$, we have for each $x \in E$ that

$$
r\left(x_{r}\right) \leqq m \cdot\left\|u\left(x_{r_{1}}\right)\right\|_{p}
$$

since $x_{r}$ is the image of $x_{r_{1}}$ under the natural mapping from $E_{r_{1}}$ 个 to $E_{r} \wedge$. Consequently if $t$ denotes the composition of the natural mapping $E \rightarrow E_{r_{1}} \hat{}$ with $u: E_{r_{1}}{ }^{\wedge} \rightarrow l^{p}$, we see that $r(x)=r\left(x_{r}\right) \leqq m \cdot\left\|u\left(x_{r_{1}}\right)\right\|_{p}=m \cdot\|t x\|_{p}$.

COROLlary 1.6. ( $p=2$.) If $E$ is a nuclear space, there exists a family of positive semidefinite Hermitian forms $\left\{(,)_{\alpha}\right\}_{\alpha \in A}$ for which the seminorms $\left\{x \rightarrow(x, x)_{\alpha}^{1 / 2}\right\}$ generate the topology of $E .(p=1$.$) If E$ is a nuclear space, there exists a family of seminorms generating the topology of $E$ of the form

$$
X \rightarrow \sum_{i \in N} \lambda_{i, \alpha}\left|\left\langle x, x_{i, \alpha}^{\prime}\right\rangle\right|
$$

where for each $\alpha$ in some index set $A, \sum_{i \in N} \lambda_{i, \alpha}<\infty$ and $\left\{x_{i, \alpha}\right\}_{i \in N}$ is an equicontinuous subset of $E^{\prime}$.

It is easy to see that if $E, F, G$ and $H$ are Banach spaces, $t: E \rightarrow F$ is linear continuous, $u: F \rightarrow G$ is nuclear, and $v: G \rightarrow H$ is linear continuous, then $u t$, $v u$, and $v u t$ are all nuclear, whence it follows readily that

Corollary 1.7. If $\left\{(,)_{\alpha}\right\}_{\alpha \in A}$ is a set of positive semidefinite Hermitian forms generating the topology of a nuclear space $E$ as in 1.6 above, then there 
exists a cofinal (in the usual ordering of the seminorms $x \rightarrow(x, x)_{\alpha}{ }^{1 / 2}$ ) subset with the property that if $(x, x)_{\alpha} \leqq k^{2} \cdot(x, x)_{\beta}$ for all $x \in E$, then the natural mapping from $E_{\beta}{ }^{\wedge}$ to $E_{\alpha}{ }^{\wedge}$ (both of which are Hilbert spaces under naturally induced inner products) is nuclear.

\section{Consequences of equicontinuity of spectral measures.}

Proposition 2.1. Let $E$ be a locally convex space and $(X, \Im, \mu)$ an equicontinuous spectral measure triple. Then the set

$$
\left\{\int f d \mu \mid f \subseteq \text {-measurable, } 0 \leqq|f| \leqq 1\right\} \subseteq \mathscr{L}(E)
$$

is also equicontinuous.

Proof hinges on the following elementary lemma whose proof is left to the reader.

LEMMA 2.2. If $(X, \Im, v)$ is a finite complex-valued measure space, then

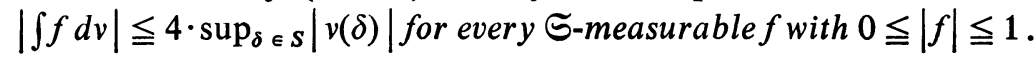

Proof of the proposition. Let $W$ be a given closed circled convex neighborhood of zero in $E$, and find a neighborhood $V$ of zero for which $\mu(\delta)[V] \subseteq 1 / 4 \cdot W$ for all $\delta \in \mathfrak{S}$. If $x^{\prime} \in E^{\prime}$ is in the polar $W^{0}$ of $W$, then for any $x \in V$ we have $\left|\left\langle\mu(\delta) x, x^{\prime}\right\rangle\right| \leqq 1 / 4$ by definition of polar. Hence

$$
\left|\left\langle\int f d \mu(x), x^{\prime}\right\rangle\right|=\left|\int f d\left\langle\mu(\cdot) x, x^{\prime}\right\rangle\right| \leqq 4 \cdot \sup _{\delta \in \mathcal{S}}\left|\left\langle\mu(\delta) x, x^{\prime}\right\rangle\right| \leqq 1
$$

for any S-measurable function $f$ with $0 \leqq|f| \leqq 1$; consequently, by the bipolar theorem, $\int f d \mu(x) \in W$. Since the choice of $V$ depended only on $W$ and $x \in V$ was arbitrary, the proposition is proved.

Proposition 2.3. Let $E[\mathfrak{I}]$ be a locally convex space, $(X, \mathfrak{S}, \mu)$ an equicontinuous spectral measure triple in $\mathscr{L}(E)$, and $\left\{V_{\alpha}\right\}$ a $\mathfrak{I}$-neighborhood basis of $0 \in E$ consisting of closed circled convex sets. Then there exists another neighborhood basis $\left\{W_{\alpha}\right\}$ of zero consisting of closed circled convex sets indexed with the same set as $\left\{V_{\alpha}\right\}$ and having the properties:

(1) if $f$ is $\mathfrak{S}$-measurable and $|f| \equiv 1$, then $\left(\int f d \mu\right)\left[W_{\alpha}\right]=W_{\alpha}$;

(2) if $f$ and $g$ are bounded $\subseteq$-measurable functions, $0 \leqq f \leqq g$, then

$$
\left(\int f d \mu\right)\left[W_{\alpha}\right] \subseteq\left(\int g d \mu\right)\left[W_{\alpha}\right]
$$

for each $W_{\alpha}$.

Proof. For each index $\alpha$ let $W_{\alpha}=\bigcap_{\{f|| f \mid \leqq 1\}}\left(\int f d \mu\right)^{-1}\left[V_{\alpha}\right]$. Clearly each $W_{\alpha}$ is closed, circled, convex and contained in $V_{\alpha}$. On the other hand, by the 
equicontinuity proved in Proposition 2.1, each $W_{\alpha}$ is a neighborhood of zero. Thus $\left\{W_{\alpha}\right\}$ is a neighborhood basis for zero in $E[\mathfrak{T}]$.

We now make a definition: a neighborhood basis for zero in $E[\mathfrak{I}]$ which satisfies (1) and (2) above will be said to be compatible with the spectral measure triple $(X, \Im, \mu)$.

The property of compatibility for a neighborhood basis of zero can of course be phrased in terms of its associated family of seminorms: using the standard relations between closed convex sets and their Minkowski (gauge) functionals, if $q_{\alpha}$ is the gauge of $W_{\alpha}$ it is easily seen that (1) and (2) are equivalent respectively to the following conditions on the seminorms $q_{\alpha}$ :

(1a) if $f$ is $\mathbb{S}$-measurable and $|f| \equiv 1$, then for all $x \in E$

$$
q_{\alpha}\left(\int f d \mu(x)\right)=q_{\alpha}(x)
$$

(2a) if $f$ and $g$ are bounded $\subseteq$-measurable functions, $0 \leqq f \leqq g$, then

$$
q_{\alpha}\left(\int f d \mu(x)\right) \leqq q_{\alpha}\left(\int g d \mu(x)\right) \text { for all } x \in E
$$

for all the seminorms $\left\{q_{\alpha}\right\}$. Seminorms with these properties will be called compatible with $(X, \mathfrak{S}, \mu)$. Notice that if $E$ is a locally convex space whose topology can be defined by a family of norms, then the topology of $E$ can be defined by a family of norms compatible with $(X, \mathfrak{S}, \mu)$.

Proposition 2.4. Let $E$ be a locally convex space, $(X, \mathcal{S}, \mu)$ an equicontinuous spectral measure triple in $\mathscr{L}(E)$. For each compatible seminorm $q$ continuous on $E$, the closed subspace $q^{-1}[0] \subseteq E$ is invariant under the spectral algebra associated with $(X, \mathfrak{S}, \mu)$, so that the spectral algebra defines an algebra of linear transformations on the quotient space $E_{q}$. The elements of the induced algebra of transformations are continuous with respect to the norm which $q$ induces on $E_{q}$ and hence may be extended uniquely by continuity to the completion $E_{q} \hat{}$, which is a Banach space under the natural extension of the norm induced on $E_{q}$ by $q$ (which we again call $q$ ). The idempotent-valued set function $\delta \rightarrow(\mu(\delta))_{q} \in \mathscr{L}\left(E_{q}{ }^{\hat{}}\right)$ is countably additive in the strong operator topology of $\mathscr{L}\left(E_{q}{ }^{\wedge}\right)$; denoting this spectral measure by $\mu_{q}$, the spectral algebra associated with $\left(X, \Im, \mu_{q}\right)$ is precisely the algebra of linear transformations induced by the spectral algebra on $E$ associated with $(X, \mathfrak{S}, \mu)$. The map $x \rightarrow x_{q}$ from $E \rightarrow E_{q}{ }^{\wedge}$ is a homomorphism of modules over the bounded $\mathfrak{S}$-measurable functions, with $f \in M$ acting as $\int f d \mu$ on $E$ and as $\int f d \mu_{q}$ on $E_{q}^{\wedge}$; i.e., $\left(\int f d \mu(x)\right)_{q}$ $=\int f d \mu_{q}\left(x_{q}\right)$. The norm $q$ on $E_{q}{ }^{\wedge}$ is compatible with $\left(X, \mathfrak{S}, \mu_{q}\right)$. If $p$ is another compatible seminorm on $E$ with $p(x) \leqq k \cdot q(x)$ for some positive $k \in \boldsymbol{R}$ and all $x \in E$, then the natural linear transformation which maps $E_{q}{ }^{\wedge}$ into $E_{p}{ }^{\wedge}$ is also 
a homomorphism of modules over the bounded S-measurable functions (with $f$ acting as $\int f d \mu_{q}$ and as $\int f d \mu_{p}$ respectively).

Proof. If $f$ is a bounded 5 -measurable function, then writing $f=|f| \cdot \operatorname{sgn} f$ and letting $k$ be a bound for $|f|$, conditions (1a) and (2a) give

$$
\begin{aligned}
q\left(\int f d \mu(x)\right) & =q\left(\int \operatorname{sgn} f d \mu\left(\int|f| d \mu(x)\right)\right) \\
& =q\left(\int|f| d \mu(x)\right) \leqq q\left(\int k d \mu(x)\right)=k \cdot q(x)
\end{aligned}
$$

whence $x \in q^{-1}[0]$ implies $\int f d \mu(x) \in q^{-1}[0]$. That the spectral algebra associated with $(X, \widehat{S}, \mu)$ will define a linear algebra of linear transformations on $E_{q}$ by quotient formation is an elementary algebraic fact; but the inequality just proved, together with the definition of the norm $q$ on $E_{q}{ }^{\lambda}$, shows that the induced transformations are continuous on $E_{q}$, hence may be extended by uniform continuity and density to $E_{q} \hat{\text { }}$. The set function $\delta \rightarrow(\mu(\delta))_{q}$ is equicontinuous (in fact, its values have uniform operator norm 1) on $E_{q}$ and is additive and countably additive there; but the pointwise convergence of an equicontinuous family of operators is equivalent to their pointwise convergence on a dense subset of the

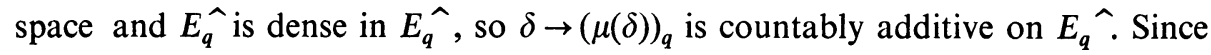
for every bounded S-measurable $f$ and every $x \in E$ (by a simple approximation argument) $\left(\int f d \mu(x)\right)_{q}=\int f d \mu_{q}\left(x_{q}\right)$, the map $x \rightarrow x_{q}$ is a homomorphism of modules over the bounded $\mathfrak{S}$-measurable functions acting on $E$ and $E_{q}{ }^{\wedge}$ as $\int f d \mu$ and $\int f d \mu_{q}$ respectively. It is easily verified that the compatibility of the norm $q$ on $E_{q}$ is inherited from that of the seminorm $q$ on $E$, whence $q$ is compatible with $\left(X, \mathfrak{S}, \mu_{q}\right)$ on $E_{q}{ }^{\wedge}$ by continuity. Again for purely algebraic reasons the natural linear mapping from $E_{q}$ to $E_{p}$ is a homomorphism of modules over the bounded 5 -measurable functions (since $q^{-1}[0]$ is a submodule of $p^{-1}[0]$ ); using continuity and the fact that $\int f d \mu_{q}\left(x_{q}\right)=\left(\int f d \mu(x)\right)_{q}$ and $\int f d \mu_{p}\left(x_{p}\right)=\left(\int f d \mu(x)\right)_{p}$ for every $x \in E$, together with the denseness of the natural image of $E$ in $E_{q}{ }^{\wedge}$ and $E_{p} \hat{}$, it is easy to see that the natural linear mapping from $E_{q} \hat{~}$ to $E_{p} \hat{~}$ is a module homomorphism with $f$ acting as $\int f d \mu_{q}$ and $\int f d \mu_{p}$ respectively.

For the class of locally convex spaces which possess a family of positive semidefinite Hermitian forms generating the topology of the space (e.g. nuclear spaces, as in 1.6 and 1.7) stronger results than those of 2.3 are available. The quotients of such spaces with respect to the null spaces of such seminorms are easily seen to be pre-Hilbert spaces in a natural way, so that the equipment of Hilbert space theory is available on their completions (in fact, such locally convex spaces are clearly dense subspaces of projective limits of Hilbert spaces - projective limits of Hilbert spaces if complete). The following proposition connects 
the spectral measure on $E$ with self-adjoint spectral measures on the quotient Hilbert spaces.

Proposition 2.5. Let $E$ be a locally convex space for which there exists a family of positive semidefinite Hermitian forms on $E$ whose associated seminorms generate the topology of $E$, and let $(X, \mathcal{S}, \mu)$ be an equicontinuous spectral measure triple in $\mathscr{L}(E)$. Then there exists a family of positive semidefinite Hermitian forms $\left\{(,)_{\alpha}\right\}$ (indexed by the same set as a given family) whose associated seminorms generate the topology of $E$ and which have the further property that for each $x$ and $y$ in $E, \delta \in \mathbb{S}$,

$$
(\mu(\delta) x, y)_{\alpha}=(x, \mu(\delta) y)_{\alpha} .
$$

Proof. The pseudo-unitary group $\mathfrak{U}=\left\{\int f d \mu \mid f \mathfrak{S}\right.$-measurable, $\left.|f| \equiv 1\right\}$ associated with $(X, \mathfrak{S}, \mu)$ possesses an invariant mean, i.e. a linear functional $m$ on the Banach space $B(\mathfrak{U})$ of bounded functions (in the uniform norm) on $\mathfrak{U}$, with $m$ positive, of norm 1 , and having the property that for every $f \in B(\mathfrak{U})$ and every $v \in \mathfrak{U}, m[f(\cdot)]=m[f(v \cdot)]$ (translation-invariance). (This follows from [6, Vol. I, p. 115] with the discrete topology on G.) Let $\left\{[,]_{\alpha}\right\}$ be a family of positive semidefinite Hermitian forms whose associated seminorms $\left\{x \rightarrow[x, x]_{\alpha}^{1 / 2}\right\}$ generate the topology of $E$. By the Schwarz inequality for the $\left\{[,]_{\alpha}^{1 / 2}\right\}$, we have

$$
\left|[u x, u y]_{\alpha}\right| \leqq[u x, u x]_{\alpha}^{1 / 2} \cdot[u y, u y]_{\alpha}^{1 / 2},
$$

and since $\mathfrak{U}$ is an equicontinuous subset of $\mathscr{L}(E)$ (by 2.1) there exists an index $\beta$, depending on $\alpha$, such that $\left|[u x, u y]_{\alpha}\right| \leqq k^{2} \cdot[x, x]_{\beta}^{1 / 2} \cdot[y, y]_{\beta}^{1 / 2}$ for a suitable $k \geqq 0$. Thus for each pair $x, y$ in $E$ and each index $\alpha,[u x, u y]_{\alpha}$ depends boundedly on $u \in \mathfrak{U}$ and hence one can form the mean $m_{u}\left([u x, u y]_{\alpha}\right)$, which we will denote by $(x, y)_{\alpha}$. It follows easily from the linearity and positivity of $m$ that the number $(x, y)_{\alpha}$ depends in a positive semidefinite Hermitian manner on $x$ and $y$. The seminorms $\left\{x \rightarrow(x, x)_{\alpha}^{1 / 2}\right\}$ generate a coarser topology than the given topology on $E$, since by the equicontinuity of $\mathfrak{U}$, given any index $\alpha$ there exists an index $\beta$ such that for all $x \in E$ and $u \in \mathfrak{U},[u x, u x]_{\alpha} \leqq k^{2} \cdot[x, x]_{\beta}$; applying the mean and using its positivity we see that $(x, x)_{\alpha}^{1 / 2} \leqq k \cdot[x, x]_{\beta}^{1 / 2}$. On the other hand, they also generate a finer topology: replacing $x$ by $u^{-1} y$ in the above we have $[y, y]_{\alpha} \leqq k^{2} \cdot\left[u^{-1} y, u^{-1} y\right]_{\beta}$ for all $u^{-1} \in \mathfrak{U}$, hence as before we have $[y, y]_{\alpha}^{1 / 2} \leqq k \cdot(y, y)_{\beta}^{1 / 2}$ for all $y \in E$. The topologies are thus identical. That $\mu$ is "self-adjoint" with respect to each $(,)_{\alpha}$ follows by the standard relation between projections and involutions: each $u \in \mathfrak{U}$ is "unitary" with respect to each of the $(,)_{\alpha}$ by translation-invariance of $m$, because

$$
\begin{aligned}
(u x, y)_{\alpha} & =m_{v}([v u x, v y])_{\alpha}=m_{v}\left(\left[v u x, v u\left(u^{-1} y\right)\right]_{\alpha}\right) \\
& =m_{w}\left(\left[w x, w\left(u^{-1} y\right)\right]_{\alpha}\right)=\left(x, u^{-1} y\right)_{\alpha}
\end{aligned}
$$


for each $x$ and $y$ in $E$ and index $\alpha$; since for each $\delta \in \mathfrak{S}$ we have $2 \mu(\delta)-1 \in \mathfrak{U}$, and since that linear transformation is its own inverse, it follows readily that $(\mu(\delta) x, y)_{\alpha}=(x, \mu(\delta) y)_{\alpha}$ for each $x$ and $y$ in $E$ and index $\alpha$.

It is easy to see that the seminorms $(\cdot, \cdot)_{\alpha}^{1 / 2}$ are compatible with $(X, \subseteq, \mu)$ in the sense of the definition following Proposition 2.3; condition (1a) of that definition is satisfied as a consequence of the translation invariance of the mean used to define the $(,)_{\alpha}$, while if $0 \leqq f \leqq g$ are bounded S-measurable functions, then $0 \leqq f^{2} \leqq g^{2}$, and it is easily verified (since the statement is clear for simple functions, it follows for all bounded $\subseteq$-measurable functions by taking limits) that

$$
\left(\int f d \mu(x), \int f d \mu(x)\right)_{\alpha}=\left(\int f^{2} d \mu(x), x\right)_{\alpha}=\int f^{2} d(\mu(\cdot) x, x)_{\alpha}
$$

so that condition (2a) follows from the positivity of the measures $(\mu(\cdot) x, x)_{\alpha}$. In addition to all the conclusions of 2.4 holding for the natural mappings of $E$ into the completions of the quotient spaces of $E$ with respect to the null spaces of these seminorms, it is clear that these complete spaces are Hilbert spaces under the inner products induced by the corresponding forms $(,)_{\alpha}$ (see e.g. [6, Chapter V]) and that the induced spectral measures $\left(X, \mathfrak{S}, \mu_{\alpha}\right)$ are self-adjoint with respect to these inner products. The last conclusion of 2.4 , however, admits the further extension:

Proposition 2.6. Let $E$ be a locally convex space, $(X, \mathfrak{S}, \mu)$ an equicontinuous spectral measure triple in $\mathscr{L}(E)$, and suppose that the topology of $E$ is generated by the seminorms $\left\{x \rightarrow(x, x)_{\alpha}^{1 / 2}\right\}$ associated with a family of positive semidefinite Hermitian forms $\left\{(,)_{\alpha}\right\}$ with the property that $(\mu(\delta) x, y)_{\alpha}=(x, \mu(\delta) y)_{\alpha}$ for all $x$ and $y$ in $E$ and all indices $\alpha$, as in 2.5 above. Suppose that for some $k \geqq 0$ $(x, x)_{\alpha} \leqq k^{2} \cdot(x, x)_{\beta}$ for all $x \in E$, so that there is a natural mapping from the

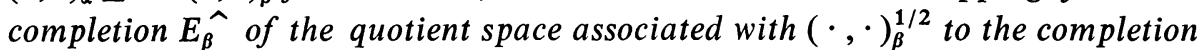
$E_{\alpha} \hat{\text { of }}$ the quotient space associated with $(\cdot, \cdot)_{\alpha}^{1 / 2}$; let $T$ denote this natural mapping. $E_{\beta} \hat{a}$ and $E_{\alpha} \hat{a}$ are Hilbert spaces under the inner products induced by $(,)_{\beta}$ and $(,)_{\alpha}$ respectively. Let $P$ denote the positive operator on $E_{\beta} \hat{\beta}$ induced by requiring that $(T x, T y)_{\alpha}=(P x, y)_{\beta}$ for all $x$ and $y$ in $E_{\beta} \hat{.}$ Let $\mu_{\beta} \hat{\wedge}$ and $\mu_{\alpha}$ denote the (self-adjoint) spectral measures induced on $E_{\beta} \hat{a n d} E_{\beta} \hat{~}$ by $\mu$. Then $P$ commutes with the values of $\mu_{\beta}$, hence with the spectral algebra associated with $\left(X, \Im, \mu_{\beta}\right)$.

Proof. The existence of $P$ under these circumstances is a well-known fact in the theory of Hilbert spaces. To prove the commutativity assertion it suffices to consider only the elements $x_{\beta}, y_{\beta}$ in $E_{\beta} \widehat{ }$ which are elements of $E_{\beta}$, i.e. elements of the image of $E$ under the canonical mapping $E \rightarrow E_{\beta}{ }^{\wedge}$, since these elements

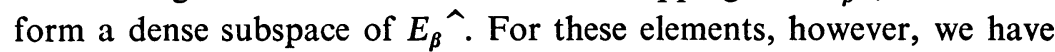




$$
\begin{aligned}
\left(P \mu_{\beta}(\delta) x_{\beta}, y_{\beta}\right)_{\beta} & =\left(T \mu_{\beta}(\delta) x_{\beta}, T y_{\beta}\right)_{\alpha}=\left(\mu_{\alpha}(\delta) x_{\alpha}, y_{\alpha}\right)_{\alpha} \\
& =\left(x_{\alpha}, \mu_{\alpha}(\delta) y_{\alpha}\right)_{\alpha}=\left(T x_{\beta}, T_{\mu_{\beta}}(\delta) y_{\beta}\right)_{\alpha} \\
& =\left(P x_{\beta}, \mu_{\beta}(\delta) y_{\beta}\right)_{\beta}=\left(\mu_{\beta}(\delta) P x_{\beta}, y_{\beta}\right)_{\beta} .
\end{aligned}
$$

Consequently, $P \cdot \mu_{\beta}(\delta)=\mu_{\beta}(\delta) \cdot P$ holds on a dense subset of $E_{\beta} \hat{~}$; by continuity, it holds on all $E_{\beta} \hat{}{ }^{\hat{n}}$. The commutativity of $P$ with the spectral algebra associated with $\mu_{\beta}$ may now be deduced by integration.

It is well known that a complete locally convex space $E$ can be represented as the projective limit of the spaces $E_{p} \hat{~ w h e r e ~} p$ runs through a directed (under the usual ordering of seminorms) family $\boldsymbol{P}$ of continuous seminorms on $E$ which generate the topology of $E$, i.e. $E=\operatorname{proj} \lim _{p} E_{p} \widehat{~}$. This projective limit space

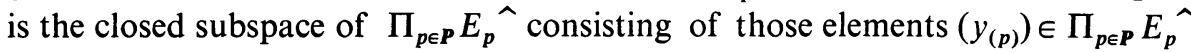
which have the following property: whenever $r$ is a finer seminorm than $q, r$ and $q$ in $\boldsymbol{P}$, then $A_{q r} y_{(r)}=y_{(q)}$, where $A_{q r}: E_{r} \hat{\imath} E_{q} \hat{}$ is the natural linear mapping; these natural mappings have the property $A_{p q} A_{q r}=A_{p r}$. These facts are discussed in [12, p. $231 \mathrm{ff}$.$] . One can define a continuous linear mapping on$ proj $\lim _{\boldsymbol{P}} E_{p}{ }^{\wedge}$ by giving a family $\left\{T_{p}\right\}_{p \in \boldsymbol{P}}$ with $T_{p} \in \mathscr{L}\left(E_{p} \hat{}\right)$ for which the obvious "commutative diagram" condition $T_{p} A_{p q}=A_{p q} T_{q}$ is satisfied for all pairs $p, q$ in $\boldsymbol{P}$ with $q$ finer than $p$, because clearly the transformation $T:\left(y_{(p)}\right) \rightarrow T_{p}\left(y_{(p)}\right)$ belongs to $\mathscr{L}\left(\Pi_{p \in \mathbf{P}} E_{p}{ }^{\wedge}\right)$ and leaves proj $\lim _{p} E_{p}{ }^{\wedge}$ invariant; we call the restriction of $T$ to proj $\lim _{\boldsymbol{P}} E_{p} \hat{}$ the projective limit of $\left\{T_{p}\right\}_{p \in P}$ and denote it by proj $\lim _{P} T_{p}$. Given a set $X$, a $\sigma$-algebra $\subseteq$ of subsets of $X$, and for each $p \in P$ a spectral measure $\mu_{p}: \Im \rightarrow \mathscr{L}\left(E_{p} \hat{}\right)$ such that $\mu_{p}(\delta) A_{p q}=A_{p q} \mu_{q}(\delta)$ for every $\delta \in \mathfrak{S}$ and pair $p$ and $q$ in $\boldsymbol{P}$ with $q$ finer than $p$, one can define a set function $\mu: \Im \rightarrow \mathscr{L}(E)$ by setting $\mu(\delta)=\operatorname{proj} \lim _{\boldsymbol{P}} \mu_{p}(\delta)$ for $\delta \in \mathfrak{S}$. That $\mu$ is a spectral measure is easily verified; call it the projective limit of the $\left\{\mu_{p}\right\}_{p \in \mathbb{P}}, \mu=\operatorname{proj} \lim _{\boldsymbol{P}} \mu_{p}$. If each $\mu_{p}$ is equicontinuous, then the mappings $\left(y_{(p)}\right) \rightarrow\left(\mu_{p}(\delta) y_{(p)}\right)$ are equicontinuous in

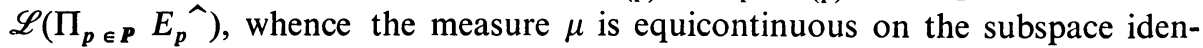
tified with $E$.

With these definitions, Propositions 2.3 through 2.6 above may be summarized in

THEOREM 2.7. Let $E$ be a complete locally convex space, $(X, \Im, \mu)$ an equicontinuous spectral measure triple in $\mathscr{L}(E)$. Then there exists a cofinal directed family $\boldsymbol{Q}$ of compatible seminorms for which, when $E$ is represented as proj $\lim _{\boldsymbol{Q}}$ $E_{q}{ }^{\hat{}}$, we have $\mu=$ proj $\lim _{\mathbf{Q}} \mu_{q}$, the $\mu_{q}: \subseteq \rightarrow \mathscr{L}\left(E_{q}{ }^{\hat{}}\right)$ being the spectral measures induced as in 2.4 above. If the topology of $E$ is induced by the seminorms associated with some family $\left\{[,]_{\alpha}\right\}$ of positive semidefinite Hermitian forms on $E$, then the $\left\{E_{q}{ }^{\wedge}\right\}_{q \in Q}$ can be taken to be Hilbert spaces and the $\left\{\mu_{q}\right\}_{q \in Q}$ to be selfadjoint-projection-valued. Finally, if $E$ is a nuclear space, the $\left\{E_{q} \hat{\}_{q \in Q}}\right.$ can be taken so that the linking mappings $A_{r q}: E_{q} \widehat{ } \rightarrow E_{r} \widehat{\wedge}$ are nuclear.

The modifications necessary when $E$ is not complete are straightforward and may be carried out by the reader; cf. [12]. 
We can now give a proof of the following proposition, announced in [18]. The result will also be seen to be a corollary of 4.1 , but the proof for this special case requires only a few geometric facts about Hilbert spaces.

Proposition 2.8. Let $E$ be a complete nuclear space, $(C, \mathfrak{B}, v)$ a spectral measure triple in $\mathscr{L}(E)$ with $\mathfrak{B}$ the Borel sets of $\boldsymbol{C}$ and $v$ an equicontinuous spectral measure of compact support in C. Let $t=\int z d v$; then $t=\sum_{\lambda \in \pi(t)} \lambda v(\{\lambda\})$, the sum meant in the sense of the limit of the net of finite subsums in the strong operator topology. (Note that any spectral operator with bounded spectrum can be so represented for suitable $v ; c f$. [17].)

Proof. As shown in [17], $\pi(t)$ consists precisely of those points $\lambda \in \sigma(t)$ for which $v(\{\lambda\}) \neq 0$. The sum $\sum_{\lambda \in \pi(t)} v(\{\lambda\})$ is convergent in the strong operator topology, because when $E$ is represented as proj $\lim _{\mathbf{Q}} E_{q}$ and $v$ as proj $\lim _{\mathbf{Q}} v_{q}$ where each $E_{q}{ }^{\wedge}$ is a Hilbert space and each $v_{q}$ self-adjoint, the sums $\sum_{\lambda \in \pi(t)} v_{q}(\{\lambda\})$ converge as sums of disjoint self-adjoint projections; thus the finite subsums of $\sum_{\lambda \in \pi(t)} v(\{\lambda\})$ form a Cauchy net. Clearly this net converges to a projection $e$ which commutes with all values of $v$, thus with $t$. The operator $t$ restricted to $(1-e) E$ is thus a spectral operator given by the integral $\int z d v_{0}$, where $v_{0}(\delta)$ is the restriction of $v(\delta)$ to $(1-e) E$ for each $\delta \in \mathfrak{B}$. It is easy to see that this restriction has empty point spectrum; on the other hand, $(1-e) E$ is nuclear as a closed subspace of a nuclear space [9]. To show that $e=1$, then, it will suffice to show that a spectral operator on a nonzero nuclear space has nonempty point spectrum, i,e. the following

LEMMA 2.9. If $t=\int z d v$ satisfies the hypotheses of 2.8 , then $t$ has nonempty point spectrum, $E \neq\{0\}$.

This in turn requires an elementary

Le MMA 2.10. Let $H_{1}$ and $H_{2}$ be Hilbert spaces, $(,)_{1}$ and $(,)_{2}$ their respective inner products; let $\mathrm{T}: \mathrm{H}_{1} \rightarrow \mathrm{H}_{2}$ be a continuous linear mapping, $P$ the positive semidefinite operator on $H_{1}$ defined by $(P x, y)_{1}=(T x, T y)_{2}$. Then $P$ is compact if and only if $T$ is.

Proof. It suffices to show that if $x_{1}, x_{2}, \cdots$ is a sequence in $H_{1}$ with $\left\|x_{i}\right\| \leqq 1$ for all $i$, then $P x_{1}, P x_{2}, \cdots$ converges if and only if $T x_{1}, T x_{2}, \cdots$ does. But

and

$$
\begin{aligned}
\left\|T x_{m}-T x_{n}\right\|_{2}^{2} & =\left(T\left(x_{m}-x_{n}\right), T\left(x_{m}-x_{n}\right)\right)_{2} \\
& =\left(P\left(x_{m}-x_{n}\right), x_{m}-x_{n}\right)_{1} \leqq 2 \cdot\left\|P x_{m}-P x_{n}\right\|_{1}
\end{aligned}
$$

$$
\begin{aligned}
\left\|P x_{m}-P x_{n}\right\|_{1}^{2} & =\left(P\left(x_{m}-x_{n}\right), P\left(x_{m}-x_{n}\right)\right)_{1} \\
& =\left(T\left(x_{m}-x_{n}\right), T P\left(x_{m}-x_{n}\right)\right)_{2} \leqq 2 \cdot\|T\| \cdot\|P\| \cdot\left\|T x_{m}-T x_{n}\right\|_{2} .
\end{aligned}
$$


Proof of 2.9. If $t=0$ we're finished; otherwise, let $x \in E$ be chosen with $t x \neq 0$, let $(,)_{2}$ be a positive semidefinite Hermitian form on $E$ which is compatible with $(C, \mathfrak{B}, v)$ and nonzero on $x$ and on $t x$, and let $(,)_{1}$ be another such form with $(y, y)_{2} \leqq k^{2} \cdot(y, y)_{1}$ for all $y \in E$ and with the natural mapping $T$ from the associated (completed) Hilbert space $H_{1}$ of $(,)_{1}$ to the associated (completed) Hilbert space $\mathrm{H}_{2}$ nuclear, thus a fortiori compact. By 2.10 , if $P \in \mathscr{L}\left(H_{1}\right)$ is defined by $(P y, z)_{1}=(T y, T z)_{2}$, then $P$ is compact. By $2.6, P$ commutes with the self-adjoint spectral measure $v_{1}$ induced on $H_{1}$ by $v$, and hence with $\int z d v_{1}$. If $x_{1}$ and $x_{2}$ are the images of $x$ in $H_{1}$ and $H_{2}$ respectively, then

$$
\left(\left[\int z d v_{2}\left(x_{2}\right)\right],\left[\int z d v_{2}\left(x_{2}\right)\right]\right)_{2}=\left(P\left[\int z d v_{1}\left(x_{1}\right)\right],\left[\int z d v_{1}\left(x_{1}\right)\right]\right)_{1}
$$

and since the left side of this is nonzero by choice of $x$ and $(,)_{2}, P \cdot \int z d v_{1} \neq 0$. Now by a standard theorem on self-adjoint operators on Hilbert spaces, $\int z d v_{1}$ also commutes with the spectral projections of the self-adjoint spectral measure which belongs to $P$; but because $P$ is compact, the spectrum of $P$ consists of countably many points all of which are isolated with the possible exception of zero, and the value of the self-adjoint spectral measure belonging to $P$ at each point in the spectrum of $P$ with the possible exception of zero is a self-adjoint projection of finite rank. (See [13, p. $227 \mathrm{ff}$. ].) The range of such a projection and its orthogonal complement reduce $\int z d v_{1}$, and the restrictions of the operator $\int z d v_{1}$ to the ranges of these projections of finite rank cannot all be zero, since $P \cdot \int z d v_{1} \neq 0$. Hence $\int z d v_{1}$ has a nonzero reducing subspace of finite dimension on which its restriction is nonzero, hence possesses an eigenvector there, consequently possesses a nonzero eigenvalue, say $\lambda_{0}$. But then $v_{1}\left(\left\{\lambda_{0}\right\}\right) \neq 0$ and thus $v\left(\left\{\lambda_{0}\right\}\right) \neq 0$, which is equivalent to $\lambda_{0}$ being an eigenvalue of $\int z d v$.

3. Induced order structures on cyclic subspaces and a representation theorem. Throughout the following, let $(X, \mathfrak{S}, \mu)$ be a fixed equicontinuous spectral measure triple on a locally convex space $E$, which will be assumed to be at least boundedly complete. For each $x \in E, \mathfrak{M}(x)$ and $\mathfrak{M}_{R}(x)$ denote the cyclic subspace and real cyclic subspace generated by $x$ (respectively). Let $\left\{q_{\alpha}\right\}_{\alpha \in A}$ be a directed family of compatible seminorms generating the topology of $\mathfrak{M}(x)$, hence of $\mathfrak{M}_{R}(x)$ : if $E$ is an $\mathscr{F}$-space, so is $\mathfrak{M}(x)$; if $E$ is an $s \mathscr{L} \mathscr{F}$-space, then $\mathfrak{M}(x)$ is generated by a bounded subset of $E$ (by the equicontinuity of the values of the spectral measure) and thus is a subspace of one of the limitands of $E$; thus in either of these cases $\mathfrak{M}(x)$ is an $\mathscr{F}$-space, and we may assume that the compatible seminorms chosen form an increasing sequence indexed by the natural numbers, $\left\{q_{n}\right\}_{n \in N}$.

We now fix the generator $x \in E$ until further notice. Let $M$ denote the set of bounded $\Im$-measurable functions (complex-valued) on $X, M_{R}$ denote the bounded $\mathfrak{S}$-measurable real-valued functions; these carry natural (pointwise) vector space and algebra operations and lattice ordering. Let $\psi: M \rightarrow \mathfrak{M}(x)$ be the map- 
ping given by $f \rightarrow \int f d \mu(x), M(x)=\psi[M], M_{R}(x)=\psi\left[M_{R}\right]$, and $N=\operatorname{ker} \psi$. $N \cap M_{R}$ is a lattice ideal in $M_{R}$, so $M_{R}(x)$ in addition to being algebraically isomorphic to $M_{R} / N$ acquires an induced lattice structure. The relations

and

$$
q_{\alpha}\left(\int f d \mu(x)\right)=q_{\alpha}\left(\int|f| d \mu(x)\right)
$$

$$
0 \leqq f \leqq g \Rightarrow q_{\alpha}\left(\int f d \mu(x)\right) \leqq q_{\alpha}\left(\int g d \mu(x)\right)
$$

insure that the image under $\psi$ of the positive cone in $M_{R}$ is normal and the lattice operations continuous, and clearly $\mu(\delta) x$ belongs to this cone for any $\delta \in \mathfrak{S}$. When $E$ is complete the lattice operations in $M_{R}(x)$ can be extended to $\mathfrak{M}_{R}(x)$, and the absolute-value function in $M(x)$ to $\mathfrak{M}(x)$, by simple closure: specifically

Proposition 3.1. Let $\mathcal{K}(x)$ denote the closure of the positive cone $\left\{\int f d \mu(x) \mid f \in M_{R}, f \geqq 0\right\}$ in $\mathfrak{M}_{R}(x)$, and assume that $E$ is complete. Then $\mathfrak{R}(x)$ determines a lattice ordering of $\mathfrak{M}_{\boldsymbol{R}}(x)$ for which the mapping $y \rightarrow|y|$ is continuous (in fact, length-preserving with respect to each compatible seminorm) and thus the lattice operations also continuous; further, $\mathcal{\Omega}(x)$ is normal.

Proof. Since ||$f|-| g|| \leqq|f-g|$ holds on $M$, the monotonicity of the $q_{\alpha}$ on positive elements shows that the mapping $y \rightarrow|y|$ is uniformly continuous on $M(x)$. Hence the absolute value function can be extended to $\mathfrak{M}(x)$ by continuity and denseness of $M(x)$ in $\mathfrak{M}(x)$, and completeness of $\mathfrak{M}_{R}(x)$. The relation $y=|y|$ characterizes elements of the positive cone in $M_{R}(x)$ and hence characterizes elements of $\mathcal{R}(x)$. The relations

$$
f \vee g=\frac{1}{2} \cdot[f+g+|f-g|]
$$

and

$$
f \wedge g=\frac{1}{2} \cdot[f+g-|f-g|]
$$

which hold on $M_{R}$ can now be used to define functions $(y, z) \rightarrow y \vee z$ and $(y, z) \rightarrow y \wedge z$ on $\mathfrak{M}_{R}(x)$ in terms of the absolute value function already defined there, and it is easy to verify that these are in fact the supremum and infimum respectively of $y$ and $z$ with respect to the ordering whose positive cone is $\boldsymbol{A}(x)$. Finally, $\boldsymbol{\Re}(x)$ is the closure of a normal cone and thus normal.

Proposition 3.2. If $E$ is complete, $\mathfrak{M}(x)$ is the R-direct sum of $\mathfrak{M}_{\boldsymbol{R}}(x)$ and $i \cdot \mathfrak{M}_{R}(x)$.

Proof. Since $|\operatorname{Re} f| \leqq|f|$ and $|i \cdot \operatorname{Im} f| \leqq|f|$ hold on $M$, the corresponding real-linear transformations on $M(x)$ can be extended via continuity to $\mathfrak{M}(x)$. The extensions are also $\boldsymbol{R}$-linear idempotents and the $\boldsymbol{R}$-direct sum representation follows immediately. 
Thus we can define real and imaginary parts of elements of $\mathfrak{M}(x)$; the real part of $y$ is just $\operatorname{Re} y$ as defined by the result of 3.2; the imaginary part of $y$ is $\operatorname{Im} y=(-i) \cdot i \cdot \operatorname{Im} y, i \cdot \operatorname{Im} y$ being defined as in 3.2 .

We have observed that if $E$ is an $\mathscr{F}$ - or $s \mathscr{L} \mathscr{F}$-space, $\mathfrak{M}(x)$ is an $\mathscr{F}$-space. Clearly $\mathfrak{M}(x)$ can be viewed as the completion of $M(x)$ in the topology generated by the $\left\{q_{n}\right\}_{n \in N}$; the following propositions are devoted to representing the completion of $M(x)$ as a space of classes of $\mathfrak{S}$-measurable functions, as in the usual construction of the $L^{1}$ space of a scalar nonnegative measure. We state for the most part only definitions and propositions, indicating how the customary proofs for scalar measures (as found in e.g. [10]) may be modified to suit this situation.

Definitions 3.3. $\delta \in \subseteq$ is null if $\mu(\delta) x=0$; "almost everywhere" in $X$ means except on a null set in $\subseteq$. Observe that $\delta \subseteq \varepsilon \Rightarrow \mu(\delta) x \leqq \mu(\varepsilon) x$ in $\mathfrak{M}_{R}(x)$ for $\delta, \varepsilon$ in $\widetilde{S}$. A sequence $\left\{f_{n}\right\}_{n \in N}$ converges to $f$ in measure or is Cauchy in measure if for every $0<\varepsilon \in \boldsymbol{R}$,

$$
\lim _{n} \mu\left(\left\{\xi|| f(\xi)-f_{n}(\xi) \mid \geqq \varepsilon\right\}\right) x=0
$$

or

$$
\lim _{m, n} \mu\left(\left\{\xi|| f_{n}(\xi)-f_{m}(\xi) \mid \geqq \varepsilon\right\}\right) x=0
$$

respectively; the sequence converges to $f$ almost uniformly if for every zeroneighborhood $V$ in $\mathfrak{M}(x)$ there exists $\delta \in \mathbb{S}$ such that $\left\{f_{n}\right\}$ converges uniformly to $f$ on $X \sim \delta$ and $\mu(\delta) x \in V$.

Proposition 3.4. If $\left\{f_{n}\right\}_{n \in N}$ converges in measure to $f$, then $\left\{f_{n}\right\}_{n \in N}$ is Cauchy in measure. If $\left\{f_{n}\right\}_{n \in N}$ converges in measure to $g$ also, then $f=g$ almost everywhere.

In proving this proposition one need only observe that $\delta \rightarrow \mu(\delta) x$ is subadditive in the lattice order of $\mathfrak{M}_{R}(x)$ and recall that the normality of the cone $\mathfrak{R}(x)$ implies that when $0 \leqq z_{\alpha} \leqq y_{\alpha}$ and $y_{\alpha} \rightarrow 0$, then also $z_{\alpha} \rightarrow 0$.

Proposition 3.5. If $\left\{f_{n}\right\}_{n \in N}$ is a sequence of measurable functions which is Cauchy in measure, then there is a subsequence $\left\{f_{n_{k}}\right\}_{k \in N}$ which is almost uniformly convergent (to a measurable function $f$ ).

Here one finds an increasing sequence $\left\{n_{k}\right\}_{k \in N}$ such that

$$
n, m \geqq n_{k} \Rightarrow q_{k}\left[\mu\left(\left\{\xi|| f_{n}(\xi)-f_{m}(\xi) \mid \geqq 1 / 2^{k}\right\}\right) x\right]<1 / 2^{k}
$$

and lets $\varepsilon_{p}=\bigcup_{k \geqq p}\left\{\xi|| f_{n_{k}}(\xi)-f_{n_{k+1}}(\xi) \mid \geqq 1 / 2^{k}\right\}$. Then by the subadditivity of $\delta \rightarrow \mu(\delta) x$ with respect to the order in $\mathfrak{M}_{R}(x)$,

$$
\mu\left(\varepsilon_{p}\right) x \leqq \sum_{k \geqq p} \mu\left(\left\{\xi|| f_{n_{k}}(\xi)-f_{n_{k+1}}(\xi) \mid \geqq 1 / 2^{k}\right\}\right) x,
$$

whence $p \geqq n_{0} \Rightarrow$ 


$$
\begin{aligned}
q_{n_{0}}\left(\mu\left(\varepsilon_{p}\right) x\right) & \leqq \sum_{k \geqq p} q_{n_{0}}\left(\mu\left(\left\{\xi|| f_{n_{k}}(\xi)-f_{n_{k+1}}(\xi) \mid \geqq 1 / 2^{k}\right\}\right) x\right) \\
& \leqq \sum_{k \geqq p} q_{k}\left(\mu\left(\left\{\xi|| f_{n_{k}}(\xi)-f_{n_{k+1}}(\xi) \mid \geqq 1 / 2^{k}\right\}\right) x\right) \\
& \leqq \sum_{k \geqq p} 1 / 2^{k}=1 / 2^{p-1},
\end{aligned}
$$

and the sets $\left\{y \mid q_{n}(y)<1 / 2^{n-1}\right\}$ form a decreasing zero-neighborhood basis in $E$. For $\xi \notin \varepsilon_{p}$, clearly $j \geqq i \geqq p \Rightarrow\left|f_{n_{j}}(\xi)-f_{n_{i}}(\xi)\right|<1 / 2^{p-1}$. (Cf. [10, p. 93].)

Proposition 3.6. If $\left\{f_{n}\right\}_{n \in N}$ is a sequence of $\mathfrak{S}$-measurable functions which is Cauchy in measure, then there exists a measurable function $f$ such that $f_{n}$ converges in measure to $f$.

The proof proceeds as in the scalar case.

Proposition 3.7. If $\left\{f_{n}\right\}_{n \in N}$ is a sequence of bounded S-measurable functions for which $\lim _{n} \int f_{n} d \mu(x)$ exists (in the topology of $\mathfrak{M}(x)$ ), then $\left\{f_{n}\right\}_{n \in N}$ is Cauchy in measure and hence converges to some $\mathbb{S}$-measurable function $f$. If $\left\{g_{n}\right\}_{n \in N}$ is a sequence of bounded $\mathfrak{S}$-measurable functions for which $\lim _{n} \int g_{n} d \mu(x)$ exists and equals $\lim _{n} \int f_{n} d \mu(x)$, then if $g$ is an $\mathfrak{S}$-measurable function to which $\left\{g_{n}\right\}_{n \in N}$ converges in measure, $f=g$ almost everywhere. If $\left\{f_{n}\right\}_{n \in N}$ and $\left\{g_{n}\right\}_{n \in N}$ are two sequences of bounded S-measurable functions for which $\lim _{n} \int f_{n} d \mu(x)$ and $\lim _{n} \int g_{n} d \mu(x)$ exist, if $f$ and $g$ are limits in measure of $f_{n}$ and $g_{n}$ respectively, and if $f=g$ almost everywhere, then $\lim _{n} \int f_{n} d \mu(x)=$ $\lim _{n} \int g_{n} d \mu(x)$.

The proofs again proceed in the pattern of the counterparts for nonnegative scalar-valued measures. $\widetilde{S}$-measurable functions $h$ for which there exists a sequence of bounded $\mathcal{S}$-measurable functions $\left\{h_{n}\right\}_{n \in N}$ converging in measure to $h$ with $\left\{\int h_{n} d \mu(x)\right\}_{n \in N}$ convergent in $\mathfrak{M}(x)$ will be called strongly integrable functions, and we shall write (unambiguously, by the preceding proposition) $\int h d \mu(x)$ for the $\lim _{n} \int h_{n} d \mu(x)$. Now the symbol $\int h d \mu(x)$ has already been defined for weak integrals on p. 301 above; however, we can see quite easily that the notions of weak and strong integrability are equivalent. One way is clear: if $h$ is strongly integrable and $\left\{h_{n}\right\}_{n \in N}$ is a sequence of bounded $\mathfrak{S}$-measurable functions as above (we may assume $h$ and $h_{n}$ real-valued with no loss of generality) with $\int h d \mu(x)=$ $\lim _{n} \int h_{n} d \mu(x)$, then for each $x^{\prime} \in \mathcal{R}(x)^{\prime}$ we have $\left\langle\int h d \mu(x), x^{\prime}\right\rangle=\lim _{n}\left\langle\int h_{n} d \mu(x), x^{\prime}\right\rangle$ $=\lim _{n} \int h_{n} d\left\langle\mu(\cdot) x, x^{\prime}\right\rangle$ and that is just $\int h d\left\langle\mu(\cdot) x, x^{\prime}\right\rangle$ since the convergence of $h_{n}$ to $h$ in $\mu(\cdot) x$-measure certainly implies its convergence to $h$ in $\left\langle\mu(\cdot) x, x^{\prime}\right\rangle$ measure. Thus $\int h d \mu(x)$ in the strong sense is $\int h d \mu(x)$ in the weak sense. To see that the converse is true we let $f$ be weakly integrable with respect to $\mu(\cdot) x$, and suppose that $\left\{f_{n}\right\}_{n \in N}$ is an increasing sequence of nonnegative bounded $\subseteq$-measurable functions converging pointwise to $|f|$; then $f_{n} \cdot \operatorname{sgn} f \rightarrow f$ pointwise, and 


$$
\begin{aligned}
\lim _{n}\left\langle\int f_{n} d \mu(x), x^{\prime}\right\rangle & =\lim _{n}\left\langle\int(\operatorname{sgn} f)^{-1} d \mu\left[\int f_{n} \cdot \operatorname{sgn} f d \mu(x)\right], x^{\prime}\right\rangle \\
& =\lim _{n}\left\langle\int f_{n} \cdot \operatorname{sgn} f d \mu(x),\left[\int(\operatorname{sgn} f)^{-1} d \mu\right]^{\prime} x^{\prime}\right\rangle \\
& =\left\langle\int f d \mu(x),\left[\int(\operatorname{sgn} f)^{-1} d \mu\right]^{\prime} x^{\prime}\right\rangle \\
& =\left\langle\int(\operatorname{sgn} f)^{-1} d \mu\left[\int f d \mu(x)\right], x^{\prime}\right\rangle
\end{aligned}
$$

so that the sequence $\left\{\int f_{n} d \mu(x)\right\}$ converges weakly to an element of $\mathfrak{M}(x)$; but by the monotone convergence theorem it converges strongly, whence by the definition of strong integral it converges to the strong integral $\int|f| d \mu(x)$. It follows readily that $f$ is strongly integrable and that in fact its strong integral is the weak $\int f d \mu(x)$.

Proposition 3.8. If $\left\{f_{n}\right\}_{n \in N}$ is a sequence of integrable functions for which $\left\{\int f_{n} d \mu(x)\right\}$ converges in $\mathfrak{M}(x)$, then there exists an integrable function $f$ to which $f_{n}$ converges in measure, and $\int f d \mu(x)=\lim _{n} \int f_{n} d \mu(x)$. If $f$ and $g$ are $\Im$-measurable and integrable functions respectively and $|f| \leqq|g|$ almost everywhere, then $f$ is integrable. If $\left\{f_{n}\right\}_{n \in N}$ is a sequence of integrable functions which converges in measure to $f$, and if $g$ is an integrable function such that $\left|f_{n}\right| \leqq|g|$ almost everywhere, then $f$ is integrable and also $\lim _{n} \int f_{n} d \mu(x)=\int f d \mu(x)$.

Again these assertions can be proved in the same way customarily used for scalar measures; cf. [10, §§26-27].

From Proposition 3.7 it is already clear that any element $y \in \mathfrak{M}(x)$ can be written as $y=\int f d \mu(x)$ for some suitable integrable function $f$, and that the class of functions $g$ for which $y=\int g d \mu(x)$ is precisely the class of functions $g$ which equal $f$ almost everywhere. The set $J$ of integrable functions is evidently a vector space under the pointwise operations, the set $J_{R}$ of real-valued integrable functions a real vector lattice under the pointwise operations and order; the map $h \rightarrow \int h d \mu(x)$ clearly preserves the operations and the order, and its kernel $P$ is precisely the set of $\mathfrak{S}$-measurable functions which are zero almost everywhere (null functions); the real null functions form a lattice ideal in $J_{R}$. Since $h \rightarrow \int h d \mu(x)$ maps $J$ onto $\mathfrak{M}(x), J / P$ is (algebraically) isomorphic to $\mathfrak{M}(x)$ as a vector space, and since any positive element of $J_{R}$ is the pointwise limit of an increasing sequence of positive bounded $\mathfrak{\Xi}$-measurable functions, it is clear that $J_{R} / P_{R}$ is isomorphic to $\mathfrak{M}_{R}(x)$ as a vector lattice. It is easy to see that the absolute-value function and the real-linear idempotents $\operatorname{Re}$ and $i \cdot \operatorname{Im}$ are well defined on $J / P$ and that $\left|f^{\sim}\right| \leftrightarrow \int|f| d \mu(x)=\left|\int f d \mu(x)\right|$ and $\operatorname{Re} f^{\sim} \leftrightarrow \operatorname{Re}\left(\int f d \mu(x)\right)=\int \operatorname{Re} f d \mu(x)$ where $f^{\sim}=f+P \in J / P$. Finally one observes that the product of a null function and an $\widetilde{\subseteq}$-measurable function is again null, so that for every bounded $\subseteq$-measurable function $f$ and every $g \in J$ and $h \in P$, we have $f g \in J, f h \in P$ and so $f$ defines an algebraic endomorphism of $J / P$. But now $J / P$ and $\mathfrak{M}(x)$ are isomorphic as $M$-modules: for each $f \in M$ and $g \in J$, as is clear for $g \in M$ and follows for all $g \in J$ by taking limits, one has 
Hence

$$
(f g)^{\sim} \leftrightarrow \int f d \mu\left[\int g d \mu(x)\right]
$$

THEOREM 3.9. Let $E$ be a locally convex space, $(X, \mathfrak{S}, \mu)$ an equicontinuous spectral measure triple in $\mathscr{L}(E), x \in E$, and suppose that $\mathfrak{M}(x)$ is complete and metrizable in the relativized topology of $E$. Then $\mathfrak{M}(x)$ and $\mathfrak{M}_{R}(x)$ are isomorphic as vector spaces and $M$ - or $M_{R^{-}}$-modules with the spaces of classes of integrable $\boldsymbol{C}$ - and $\boldsymbol{R}$-valued functions (respectively) on $X$ modulo null functions, and $\mathfrak{M}_{R}(x)$ is also isomorphic as a vector lattice with the corresponding space of classes of functions; the isomorphism is given by $f^{\sim} \leftrightarrow \int f d \mu(x)$. The isomorphism also preserves absolute value (for scalars $\boldsymbol{R}$ or $\boldsymbol{C}$ ) and the formation of real and imaginary parts.

Corollary 3.10 [BADE]. Let $E$ be an $\mathscr{F}$-or s $\mathscr{L} \mathscr{F}$-space, $(X, \mathfrak{S}, \mu)$ a spectral measure triple in $\mathscr{L}(E)$, and let $\mathfrak{M}(x)$ be a cyclic subspace in $E$. Then for every $y \in \mathfrak{M}(x)$ there exists a (possibly unbounded) spectral operator associated with $(X, \mathfrak{S}, \mu)$ which carries $x$ to $y$.

In fact, it suffices to take the operator $\int f d \mu$ for any integrable function $f$ for which $y=\int f d \mu(x)$. Using the representation given by 3.9 one may also give immediate and natural proofs of the following theorems of Bade [3] (extended to the more general metrizable case from Banach spaces):

If $E$ is an $\mathscr{F}$ - or $s \mathscr{L} \mathscr{F}$-space, $(X, \Im, \mu)$ a spectral measure triple on $E$ and $x \in E$, then the restrictions of the values of $\mu$ to $\mathfrak{M}(x)$ form a complete Boolean algebra of projections, and this algebra is countably decomposable.

If $E$ is an $\mathscr{F}$ - or $s \mathscr{L} \mathscr{F}$-space, then there exists a countable set of linear forms $\left\{x_{n}^{\prime}\right\}_{n \in N} \subseteq \mathfrak{R}(x)^{\prime}$ such that $\left\langle\mu(\delta) x, x_{n}^{\prime}\right\rangle=0$ for all $n$ implies that $\mu(\delta) x=0$, whence by totality of $\{\mu(\varepsilon) x\}_{\varepsilon \in \mathcal{G}},\left.\mu(\delta)\right|_{\mathfrak{W}(x)}=0$.

NB: In the Banach-space case considered by Bade, $\mathfrak{M}(x)^{\prime}$ had equicontinuous absorbant subsets and thus one could select a single $x^{\prime} \in \mathbb{R}(x)^{\prime}$ with the property that $\left\langle\mu(\delta) x, x^{\prime}\right\rangle=\left.0 \Rightarrow \mu(\delta)\right|_{\mathfrak{M}(x)}=0$. This fails to be true in general even for Fréchet spaces: the spectral measure given by the coördinate projections on the space $\omega$ of complex-valued sequences, equipped with the topology of coördinatewise convergence, has the cyclic vector $(1,1, \cdots)$, but $\mu(\cdot) x$ is not absolutely continuous with respect to any measure $\left\langle\mu(\cdot) x, x^{\prime}\right\rangle\left(x^{\prime} \in \mathfrak{R}(x)^{\prime}\right)$ since every element of $\omega^{\prime}=\phi$ is zero on a subspace of finite codimension. In fact, one can quite easily see that if such a Bade functional exists, then $\mathfrak{M}(x)$ possesses a continuous norm, namely $y \rightarrow\left\langle|y|, x^{\prime}\right\rangle$; it is not much harder to see that such a Bade functional will exist when and only when $\mathfrak{M}(x)$ possesses a continuous norm.

The following proposition leads to generalizations of [3, Lemma 2.6 and Theorem 2.7] and shows to what extent related ideas can be carried over to arbitrary locally convex spaces. We do not require the deep relations between vector- and scalar-valued measures of [5]. 
Proposition 3.11. Let $E$ be an $\mathscr{F}$ - or $s \mathscr{L} \mathscr{F}$-space, $(X, \mathfrak{S}, \mu)$ a spectral measure triple in $\mathscr{L}(E)$, and let $H$ be a majorized subset of $\mathfrak{M}_{R}(x)$. Then the set of suprema of finite subsets of $H$, directed naturally upward under the ordering in $\mathfrak{M}_{\mathbb{R}}(x)$, is convergent and its limit is the supremum of $H$.

Proof. Assume that $H \subseteq \mathfrak{R}(x)$ with no loss of generality, and let $x_{0} \in \mathfrak{R}(x)$ majorize $H$. Any countable subset of $H$ has a supremum which is the limit of the suprema of its finite subsets, for if $H_{0} \subseteq H$ is countable we may enumerate it, say as $H_{0}=\left\{h_{n}\right\}_{n \in N}$, and let $x_{n}=h_{1} \vee \cdots \vee h_{n}$ for each $n \in N$. For each $n$ let $f_{n}$ be an integrable function with $x_{n}=\int f_{n} d \mu(x)$. For each pair $m, n$ with $1 \leqq m<n$ the set of points of $X$ at which the inequalities $0 \leqq f_{m} \leqq f_{n} \leqq f_{0}$ fail to hold is null, and there are only countably many such sets, so one may redefine the $f_{n}$ to be zero on their union without changing integrals, then let $f$ be the pointwise supremum (=limit) of the redefined $f_{n}$. By Proposition $3.8 f$ is integrable and $\lim _{n} \int f_{n} d \mu(x)=\int f d \mu(x)$. It follows easily from the facts that $\Re(x)$ is closed and normal (respectively) that $\int f d \mu(x)$ majorizes the $\left\{x_{n}\right\}_{n \in N}$ and is the smallest element of $\mathfrak{M}_{R}(x)$ with that property, since by $3.8 x_{n} \rightarrow \int f d \mu(x)$ in the topology of $E$. Since the $\left\{x_{n}\right\}_{n \in N}$ are a cofinal family of finite suprema of subsets of $H_{0}$, we have the desired result.

For the general case, let $\mathfrak{G}$ denote the family of finite subsets of $H$, directed under inclusion. Clearly $\lim _{G \in \mathfrak{G}}\left\langle\bigvee\{h \mid h \in G\}, x^{\prime}\right\rangle$ exists for each $x^{\prime} \in \mathfrak{M}_{R}(x)^{\prime}$, since the family of finite suprema is monotone, majorized, and $\mathfrak{M}_{R}(x)^{\prime}=\mathfrak{R}(x)^{\prime}$ $-\mathfrak{R}(x)^{\prime}$. The topology of $\mathfrak{M}_{R}(x)$ is that of uniform convergence on equicontinuous subsets of $\boldsymbol{R}(x)^{\prime}$, so that if the family of finite suprema fails to converge in that topology there must exist an equicontinuous $S \subseteq \Omega(x)^{\prime}$, and $\varepsilon>0$, and a cofinal family $\mathfrak{G} \subseteq \mathfrak{G}$ with the property that to each $F \in \mathfrak{G}$ there corresponds an $x_{F}{ }^{\prime} \in S$ such that

$$
\left\langle\bigvee\{h \mid h \in F\}, x_{F}^{\prime}\right\rangle \leqq \lim _{\boldsymbol{G} \in \mathfrak{S}}\left\langle\bigvee\{h \mid h \in G\}, x_{F}^{\prime}\right\rangle-\varepsilon .
$$

Select some $F_{1} \in \mathfrak{G}$. If $F_{1}, \cdots, F_{n-1}$ have been selected, choose $F_{n} \in \mathbb{G}$ such that $\left\langle\bigvee\left\{h \mid h \in F_{n}\right\}, x_{F_{n-1}}^{\prime}\right\rangle>\lim _{G \in \mathfrak{R}}\left\langle\bigvee\{h \mid h \in G\}, x_{F_{n-1}}^{\prime}\right\rangle-\varepsilon / 2$ and $F_{n} \supseteq F_{n-1}$. Now since $\bigcup_{n \in N} F_{n}$ is a countable subset of $H$, it possesses a supremum, and since the sets $\left\{F_{n}\right\}$ form a cofinal family of subsets of their union, the finite suprema $\left\{\bigvee\left\{h \mid h \in F_{n}\right\}\right\}_{n \in N}$ approach their supremum in the topology of $E$, hence uniformly on $S$. But this is contradictory, because if $n \in N$ is so large that

$$
\left\langle\bigvee\left\{h \mid h \in F_{n}\right\}, x^{\prime}\right\rangle>\lim _{m}\left\langle\bigvee\left\{h \mid h \in F_{m}\right\}, x^{\prime}\right\rangle-\varepsilon / 2
$$

uniformly for $x^{\prime} \in S$, it is still supposed to be the case that

so

$$
\begin{gathered}
\lim _{m}\left\langle\bigvee\left\{h \mid h \in F_{m}\right\}, x_{F_{n}}^{\prime}\right\rangle \geqq \lim _{G \in \mathfrak{G}}\left\langle\bigvee\{h \mid h \in G\}, x_{F_{n}}^{\prime}\right\rangle-\varepsilon / 2 \\
\left\langle\bigvee\left\{h \mid h \in F_{n}\right\}, x_{F_{n}}^{\prime}\right\rangle>\lim _{G \in \mathfrak{G}}\left\langle\bigvee\{h \mid h \in G\}, x_{F_{n}}^{\prime}\right\rangle-\varepsilon
\end{gathered}
$$


contrary to having $F_{n} \in \mathfrak{G}$.

So $\mathfrak{M}_{R}(x)$ is an order-complete vector lattice, with continuous vector lattice operations, and with the property that the suprema and infima of infinite subsets are the topological limits of the directed families suprema of and infima of finite subsets of the infinite one in question. 3.13 and 3.15 below will extend this result to nonmetrizable and in fact merely boundedly complete locally convex spaces, showing as a by-product that in this latter case cyclic subspaces are complete, even though the space $E$ may not be.

LEMMA 3.12. Let $E$ be a locally convex space which is complete, $(X, \mathfrak{S}, \mu)$ an equicontinuous spectral measure triple in $\mathscr{L}(E), q$ a continuous seminorm on $E$ compatible with $(X, \mathcal{G}, \mu)$. Let $E_{q}{ }^{\wedge}$ and $\mu_{q}$ denote as before the completion of $E / q^{-1}[0]$ and the spectral measure induced on it by $\mu$ (see 2.4). For any $x \in E$ the natural (restricted quotient) mapping of $\mathfrak{M}(x)(\subseteq E)$ into $\mathfrak{M}\left(x_{q}\right)\left(\subseteq E_{q}\right)$ preserves order, absolute value, finite lattice operations and formation of real and imaginary parts.

Proof. The assertions of the lemma are clear for elements of $E$ which have the form $\int f d \mu(x)$ for $f$ a bounded $\mathfrak{S}$-measurable function, and since the lattice operations and formation of real and imaginary parts are defined by extending continuous operations defined on dense subspaces both in $\mathfrak{M}(x)$ and $\mathfrak{M}\left(x_{q}\right)$, the lemma follows.

Proposition 3.13. Let $E$ be a complete locally convex space, $(X, \mathfrak{S}, \mu)$ an equicontinuous spectral measure triple in $\mathscr{L}(E), x \in E . \mathfrak{M}_{R}(x)$ is an ordercomplete vector lattice with the property that the supremum of an infinite set is the (topological) limit of the directed family of suprema of finite subsets.

Proof. Let $H$ be a subset of $\mathfrak{M}_{R}(x)$ with $x_{0}$ majorizing $H$. For each compatible seminorm $q$ on $E$ we have $H_{q}=\left\{h_{q} \mid h \in H\right\}$ majorized by $\left(x_{0}\right)_{q}$, and for each finite subset $G \subseteq H$ we have $(\bigvee\{h \mid h \in G\})_{q}=\bigvee\left\{h_{q} \mid h \in G\right\}$, by the lemma.

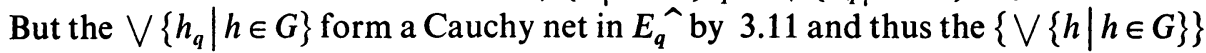
have the Cauchy property with respect to the seminorm $q$; since there are sufficiently many compatible seminorms to generate the uniformity of $E,\{\bigvee\{h \mid h \in G\}\}_{G \subseteq H}$ is a Cauchy net in $E$ and consequently convergent; since $\boldsymbol{R}(x)$ is closed and normal, the limit must be the supremum of $\boldsymbol{H}$.

Proposition 3.14. Under the hypotheses of Proposition 3.13, for each $y \in \mathfrak{R}(x)$ the net $\{n x \wedge y\}_{n \in N}$ converges to $y$.

Proof. For each compatible seminorm $q$ on $E$ we have $(n x \wedge y)_{q}=n x_{q} \wedge y_{q}$, by Lemma 3.12. Since $E_{q}{ }^{\wedge}$ is complete and metrizable, there exists a nonnegative S-measurable function $f$ for which $y_{q}=\int f d \mu_{q}\left(x_{q}\right)$, by Theorem 3.9, and $n x_{q} \wedge y_{q}=\int(n \cdot 1 \wedge f) d \mu_{q}\left(x_{q}\right)$. By the "Lebesgue dominated convergence theorem" part of Proposition 3.8, $\left\{n x_{q} \wedge y_{q}\right\}_{n \in N}$ is a sequence convergent to 
$\int f d \mu_{q}\left(x_{q}\right)=y_{q}$. Since the topology of $E$ is determined by the mappings $E \rightarrow E_{q} \hat{\text {, }}$ we have $\lim _{n}(n x \wedge y)=y$.

A result of this last proposition is that the hypothesis that $E$ be complete can be relaxed to the requirement that $E$ be merely boundedly complete. Let $E$ be a boundedly complete space, $(X, \mathfrak{S}, \mu)$ an equicontinuous spectral measure in $\mathscr{L}(E), x \in E$. Let $E^{\wedge}$ be the completion of $E$. Each spectral projection $\mu(\delta), \delta \in \mathbb{S}$, has a continuous extension to $E^{\wedge}$; these extensions, which we shall call $\mu^{\wedge}(\delta)$, are also equicontinuous, and as a result the countable additivity of $\mu \wedge(\cdot)$ on the dense subset $E \subseteq E^{\wedge}$ implies the countable additivity of $\mu^{\wedge}(\cdot)$ on all of $E^{\wedge}$. Hence the apparatus we have constructed for complete spaces (vector lattice structure, etc.) is available on $E^{\wedge}$ and on the real cyclic subspace generated by $x$ in $E^{\wedge}$, which we shall call $\mathfrak{M}_{\boldsymbol{R}} \hat{}^{\wedge}(x)$ (unambiguously, since this space is clearly a completion of $\mathfrak{M}_{\mathbb{R}}(x) \subseteq E$ ) and the cyclic subspace $\mathfrak{M}^{\wedge}(x)$. We let $\mathfrak{R}^{\wedge}(x)$ denote the positive cone in $\mathfrak{M}_{\boldsymbol{R}} \hat{}^{(x)}$; clearly it is the closure of $\mathfrak{N}(x)$ in $E^{\wedge}$. Moreover, $\mathfrak{M}_{\mathbb{R}}(x)=\mathfrak{M}_{\mathbb{R}} \hat{(x)} \cap E$ and $\mathfrak{M}(x)=\mathfrak{M}^{\wedge}(x) \cap E$, and also $\mathfrak{R}(x)=\mathfrak{R}^{\wedge}(x) \cap E$. Let $y \in \mathfrak{R}^{\wedge}(x)$; then there exists a net of bounded nonnegative $\mathcal{S}$-measurable functions $\left\{f_{\alpha}\right\}_{\alpha \in A}$ such that $y_{\alpha}=\int f_{\alpha} d \mu^{\wedge}(x)=\int f_{\alpha} d \mu(x)$ is a net converging to $y$. By the continuity of lattice operations in $\mathfrak{M}_{\boldsymbol{R}} \hat{(}(x)$, for each $n \in \boldsymbol{N}$ the net $\left\{n x \wedge y_{\alpha}\right\}_{\alpha \in A}$ converges to $n x \wedge y$. But the net $n x \wedge y_{\alpha}=\int\left(n \cdot 1 \wedge f_{\alpha}\right) d \mu(x)$ is a bounded (since the order interval $[0, n x]$ is bounded) Cauchy net of elements of $E$, hence its limit $n x \wedge y$ in $E^{\wedge}$ actually belongs to $E$. By 3.14, the sequence $\{n x \wedge y\}_{n \in N}$ converges to $y$ in $E^{\wedge}$; but this sequence in $E$ is Cauchy and thus bounded, so its limit $y$ again turns out to lie in $E$. Thus $\mathcal{R}^{\wedge}(x) \subseteq E$, and so is $\mathfrak{M}_{\boldsymbol{R}} \hat{R}^{(x)}=\mathfrak{R}^{\wedge}(x)-\mathfrak{K}^{\wedge}(x)$. Consequently, $\mathfrak{M}_{\boldsymbol{R}} \hat{(}(x)=\mathfrak{M}_{\boldsymbol{R}}(x)$. It follows that the absolute-value and $\operatorname{Re}$ and $i \cdot \operatorname{Im}$ functions on $\mathfrak{M}^{\wedge}(x)$, which would take their values in $\mathfrak{M}_{\boldsymbol{R}} \hat{\wedge}(x)$ in any event, take their values in the complete $\boldsymbol{R}$-subspace $\mathfrak{M}_{R}(x)$ of $E$ and can thus be defined on $\mathfrak{M}(x)$ just as they were in 3.2 under the assumption that $E$ was complete. Since $\operatorname{Re}$ and $i \cdot \operatorname{Im}$ represent $\mathfrak{M}(x)$ as the direct sum, internal to $E$, of the complete space $\mathfrak{M}_{R}(x)$ and a space topologically isomorphic to it, $\mathfrak{M}(x)$ is also complete. Thus

Proposition 3.15. Let $E$ be a boundedly complete space, $(X, \subseteq, \mu)$ an equicontinuous spectral measure in $\mathscr{L}(E), x \in E$. Then the cyclic subspaces $\mathfrak{M}_{\mathbb{R}}(x)$ and $\mathfrak{M}(x)$ are complete, and thus the conclusions of $3.1,3.2$ and 3.13 hold $\left({ }^{2}\right)$.

The order-completeness of these vector lattices leads to the fact that the closure of $\{\mu(\delta)\}_{\delta \in \subseteq}$ is a complete Boolean algebra of projections, the Boolean algebra ordering being again that of inclusion of ranges. This ordering and the vector lattice order on the cyclic subspaces are closely related:

(2) Results almost identical to those of $\$ 2$ and $3.1,3.2$ and 3.15 can similarly be derived for homomorphisms of algebras $\mathscr{C}(X)$ into $\mathscr{L}(E)$, where $E$ is boundedly complete and the unit ball of $\mathscr{C}(X)$ is sent to an equicontinuous family of operators. Establishing the order-completeness of the cyclic subspace lattices, however, will in general require weak sequential completeness of $E$. 
LEMMA 3.16. Every projection in the strong closure of $\{\mu(\delta)\}_{\delta \in \mathcal{G}}$ leaves each order interval $[0, x] \subseteq \mathfrak{\Omega}(x)$ in $\mathfrak{M}_{R}(x)$ invariant for each $x \in E$ (and so leaves each $\mathfrak{M}_{R}(x)$ and $\mathfrak{M}(x)$ invariant $)$. If $e$ and $f$ are two idempotents in $\mathscr{L}(E)$ which leave each order interval $[0, x] \subseteq \Re(x)$ invariant for each $x \in E$, then they

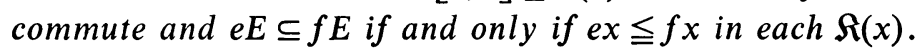

Proof. The first statement is obvious, since these intervals are closed sets which are invariant under the $\{\mu(\delta)\}_{\delta \in \mathcal{G}}$. Suppose $e$ and $f$ are two idempotents with the property described above; then for each $x \in E$ we have $0 \leqq e \leqq 1$ and $0 \leqq f \leqq 1$ in the usual ordering of operators on the partially ordered space $\mathfrak{M}_{R}(x)$. In fact, if $y \in \mathfrak{R}(x)$, then $\mathfrak{R}(y) \subseteq \mathfrak{R}(x)$ so $\mathfrak{R}(x)^{\prime} \subseteq \mathfrak{R}(y)^{\prime}$ and thus for any $x^{\prime} \in \Re(x)^{\prime}, 0 \leqq\left\langle e y, x^{\prime}\right\rangle \leqq\left\langle y, x^{\prime}\right\rangle$ and consequently $0 \leqq e y \leqq y$ in the ordering of $\mathfrak{M}_{R}(x)$; similarly for $f$. Thus for each $x \in E$, in the ordering of $\mathfrak{M}_{\mathbb{R}}(x)$ we have $e x \leqq x$, so $f e x \leqq f x$ and (similarly) $e f x \leqq e x$ by positivity; on the other hand, since $e \leqq 1$ and $f \leqq 1$ we also have $f e x \leqq e x$, ef $x \leqq f x$. Thus $f e(1-f) x \leqq f(1-f) x=0$, whence $f e x \leqq f e f x \leqq e f x$; similarly $e f x \leqq f e x$ and so $f e x=e f x$ for all $x \in E$, i.e. $e f=f e$. Now if $e E \subseteq f E$ then $e=e f=f e$, so $e x \leqq f e x=f x$ for each $x \in E$, while on the other hand if $e x \leqq f x$ in $\mathfrak{M}_{R}(x)$ for all $x \in E$, then $e x=e^{2} x \leqq e f x=f e x \leqq e x$ and so $e=e f=f e$, thus $e E \subseteq f E$.

Proposition 3.17. The strong closure of $\{\mu(\varepsilon)\}_{\varepsilon \in \mathcal{G}}$ is a complete Boolean algebra of idempotents in $\mathscr{L}(E)$.

Proof. Evidently this strong closure is composed of idempotents which are equicontinuous with the same modulus of equicontinuity as $\{\mu(\delta)\}_{\delta \in \mathcal{G}}$, which leave all order intervals $[0, x]$ invariant, and which form a Boolean algebra. If $\left\{e_{\alpha}\right\}$ is a family of idempotents in the strong closure, let $\left\{e_{\beta}\right\}$ be the family of finite suprema of these $\left\{e_{\alpha}\right\}$ - the finite Boolean operations are definable using the algebraic operations of $\mathscr{L}(E)$. The set $\left\{e_{\beta}\right\}$ is naturally directed upward, and by the lemma above this fact implies that for each $x \in E\left\{e_{\beta} x\right\}$ is directed upward and majorized in the order of $\mathfrak{M}_{R}(x)$, thus converges. Thus $\left\{e_{\beta}\right\}$ converges in the strong operator topology, surely to something in the strong closure of $\{\mu(\delta)\}_{\delta \in \Xi}$; by the lemma the range of the limit $e$ contains all of the ranges of the $\left\{e_{\beta}\right\}$; conversely if $x \in e E$ then $x=e x=\lim _{\beta} e_{\beta} x$ so $x$ is in the closure of $\bigcup_{\beta} e_{\beta} E$, and clearly $\left(\bigvee_{\beta} e_{\beta}\right) E=\left(\bigvee_{\alpha} e_{\alpha}\right) E$.

This proposition makes possible the following small extension theorem, which will be needed in $\$ 4$.

Proposition 3.18. Let $E$ be a boundedly complete locally convex space and $\left(X, \mathfrak{B}_{0}, \mu\right)$ an equicontinuous spectral measure triple where $X$ is a compact Hausdorff space and $\mathfrak{B}_{0}$ its $\sigma$-algebra of Baire subsets. Then there is one and only one Borel spectral measure triple $\left(X, \mathfrak{B}, \mu^{\sim}\right)$ which extends $\left(X, \mathfrak{B}_{0}, \mu\right)$ and is regular in the sense that 


$$
\mu^{\sim}(\varepsilon)=\lim _{\kappa} \mu \sim(\kappa)=\lim _{\zeta} \mu \sim(\zeta) \text { for each } \varepsilon \in \mathfrak{B}
$$

in the strong operator topology, where $\kappa$ runs through the compact subsets of $\varepsilon$ and $\zeta$ through the open supersets, naturally directed upward and downward respectively.

Proof. Let $\left(X, \mathfrak{B}_{0}, \mu\right)$ be given, and let $\mathfrak{C}$ denote the compact Baire sets, $\mathfrak{U}$ the open Baire sets in $X$. For each compact $\varepsilon \subseteq X$ define

$$
\lambda(\varepsilon)=\bigwedge\{\mu(\zeta) \mid \varepsilon \subseteq \zeta \in \mathfrak{U}\}
$$

the infimum being taken in the order of the complete Boolean algebra formed by the strong closure of $\{\mu(\delta)\}, \delta \in \mathfrak{B}_{0}$, and thus being a topological limit in the strong topology with the further property that

$$
\lambda(\varepsilon) x=\bigwedge\{\mu(\zeta) x \mid \varepsilon \subseteq \zeta \in \mathfrak{U}\}
$$

in the vector lattice order of $\mathfrak{M}_{R}(x)$ for each $x \in E$. Clearly these mappings $\lambda(\varepsilon), \varepsilon \subseteq X$ compact, have the property

$L:$ for each $x \in E, x^{\prime} \in \mathfrak{R}(x)^{\prime}:\left\langle\lambda(\varepsilon) x, x^{\prime}\right\rangle=\inf \left\{\left\langle\mu(\zeta) x, x^{\prime}\right\rangle \mid \varepsilon \subseteq \zeta \in \mathfrak{U}\right\}$.

Now for each open $\delta \subseteq X$ define

$$
\lambda_{*}(\delta)=\bigvee\{\lambda(\theta) \mid \delta \supseteq \theta, \theta \text { compact }\} .
$$

Again $\lambda_{*}(\delta)$ is in the strong closure of $\{\mu(\varepsilon)\}, \varepsilon \in \mathfrak{B}_{0}$, and the dual of $L$ holds. Finally, for every $\eta \cong X$ define

$$
\mu^{\sim *}(\eta)=\bigwedge\left\{\lambda_{*}(\gamma) \mid \gamma \supseteq \eta, \gamma \text { open }\right\}
$$

and the property analogous to $L$ holds. Hence by $\left[10, \S 53\right.$, Theorem E], if $\mu^{\sim}$ denotes the restriction of $\mu^{\sim *}$ to $\mathfrak{B}$, the set function $\varepsilon \rightarrow\left\langle\mu \sim(\varepsilon) x, x^{\prime}\right\rangle$ is a countably additive measure for every $x \in E$ and $x^{\prime} \in \mathfrak{R}(x)^{\prime}$. The monotone convergence Theorem 1.1 assures that $\mu^{\sim}(\cdot)$ is strongly countably additive, hence is a strongly additive idempotent-valued measure whose domain is $\mathfrak{B}$. Since each $\left\langle\mu \sim(\cdot) x, x^{\prime}\right\rangle$ is a regular Borel measure for $x^{\prime} \in \mathfrak{R}(x)^{\prime}, \mu^{\sim}$ is regular; each $\left\langle\mu^{\sim}(\cdot) x, x^{\prime}\right\rangle$ is an extension of $\left\langle\mu(\cdot) x, x^{\prime}\right\rangle$ by $[10, \S 54$, Theorem $\mathrm{D}]$ so $\mu^{\sim}$ is an extension of $\mu$. Just as in the scalar-valued case, $\mu^{\sim}$ is the only regular Borel measure whose restriction to $\mathfrak{B}_{0}$ is $\mu$; if $v$ is another such measure, so $(X, \mathfrak{B}, v)$ is a spectral measure triple, let $\mathfrak{N}_{R}(x)$ and $\mathfrak{S}(x)$ denote the real cyclic subspace and positive cone therein generated by $x$ under the action of $v$; since $v$ extends $\mu$, $\mathfrak{N}_{\mathbb{R}}(x) \supseteq \mathfrak{M}_{R}(x)$ and $\mathfrak{H}(x) \supseteq \mathfrak{R}(x)$, whence $\mathfrak{H}(x)^{\prime} \subseteq \mathfrak{R}(x)^{\prime}$. This last inclusion shows that for every $x^{\prime} \in \mathfrak{S}(x)^{\prime},\left\langle\mu \sim(\cdot) x, x^{\prime}\right\rangle$ and $\left\langle v(\cdot) x, x^{\prime}\right\rangle$ are regular positive scalar-valued Borel measures whose restrictions to the Baire sets agree, hence by $[10, \S 52$, Theorem $\mathrm{H}]$ are equal - but $E \otimes E^{\prime}$ is generated by elements of the form $x \otimes x^{\prime}, x^{\prime} \in \mathfrak{T}(x)^{\prime}$, so $\mu^{\sim}=v$. 
Since the values of $\mu \sim$ lie in the strong closure of those of $\mu$, it is clear that the moduli of continuity and cyclic subspaces of $\mu \sim$ and $\mu$ are the same.

\section{Spectral measures on Montel spaces.}

THEOREM 4.1. Let $E$ be a locally convex space whose closed bounded subsets are compact, $\left(X, \mathfrak{B}_{0}, \mu\right)$ an equicontinuous Baire spectral measure triple in $\mathscr{L}(E),\left(X, \mathfrak{B}, \mu^{\sim}\right)$ its extension to a regular Borel spectral measure. Then $\left(X, \mathfrak{B}, \mu^{\sim}\right)$ possesses atoms, hence point atoms, and the supremum (in the sense of 3.17) of these point atoms is the identity in $\mathscr{L}(E)$.

The proof requires several lemmata.

LEMMA 4.2. If $v$ is a regular (nonnegative scalar-valued) Borel measure on a compact Hausdorff space $X$ and $v$ possesses atoms, then each atom of $v$ contains a point whose measure is positive.

The proof is left to the reader.

LEMMA 4.3. Let $E$ be a boundedly complete locally convex space, $(X, \subseteq, \mu)$ an equicontinuous spectral measure triple in $E, x \in E$ and $x^{\prime} \in \mathcal{R}(x)^{\prime}$. There is a natural continuous mapping of $\mathfrak{M}(x)$ onto a dense subspace of $L^{1}\left(\left\langle\mu(\cdot) x, x^{\prime}\right\rangle\right)$, namely the extension by continuity of the mapping of classes of bounded S-measurable functions modulo $\mu(\cdot) x$-null functions to classes of bounded ऽ-measurable functions modulo $\left\langle\mu(\cdot) x, x^{\prime}\right\rangle$-null functions.

Proof. That the mapping is well defined on $M(x)$ is clear; its continuity follows by taking some compatible seminorm $q$ for which $\left|\left\langle y, x^{\prime}\right\rangle\right| \leqq k \cdot q(y)$ holds for all $y \in E$, for then

$$
\begin{aligned}
\int|f| d\left\langle\mu(\cdot) x, x^{\prime}\right\rangle=\left\langle\int|f| d \mu(x), x^{\prime}\right\rangle & \leqq k \cdot q\left(\int|f| d \mu(x)\right) \\
& =k \cdot q\left(\int f d \mu(x)\right),
\end{aligned}
$$

the last equality by the compatibility of $q$.

LEMMA 4.4. Let $(X, \subseteq, v)$ be a totally finite (non-negative, scalar) measure space. The natural injection $L^{\infty}(v) \rightarrow L^{1}(v)$ is a compact mapping if and only if $(X, \mathfrak{S}, v)$ is purely atomic.

Proof. Suppose this injection is compact. This property is inherited by the measure subspaces $\left(Y, \Im_{Y}, v\right)$ of $\left(X, S_{,} v\right)(Y \in \Im)$, so by separating $\left(X, S_{,} v\right)$ into purely atomic and purely nonatomic parts, it suffices to show that a purely nonatomic $(X, \mathfrak{S}, v)$ for which this injection is compact must have $v=0$. If this were not the case, one could assume without loss of generality that $v(X)=1$. 
For each finite partition $P$ of $X$ by elements of $\mathfrak{S}$, let $A_{P} \in \mathscr{L}\left(L^{1}\right)$ be the operator defined by $A_{P}(f)=\sum_{\varepsilon \in P}\left((1 / v(\varepsilon)) \int f \chi_{e} d v\right) \chi_{e}$ (cf. [8, p. 297]). The set $\Pi$ of such partitions is naturally directed under "fineness," and $\lim _{P} A_{P}=1$ in the strong operator topology; also, $\left\|A_{P}\right\|=1$ for all $P$. By hypothesis on the injection $L^{\infty}(v) \rightarrow L^{1}(v)$, then, $\lim _{P}\left\|A_{P} f-f\right\|_{1}=0$ uniformly for $|f| \leqq 1$, and in particular for characteristic functions of elements of $\Im$; let $P$ be so fine that $\left\|A_{P} f-f\right\|_{1}<1 / 2$ for all such $f$. For each $\varepsilon \in P$, let $\delta_{\varepsilon} \subseteq \varepsilon$ be an element of $\mathfrak{S}$ for which $v\left(\delta_{\varepsilon}\right)=(1 / 2) v(\varepsilon) ; \delta_{\varepsilon}$ exists since $(X, \Im, v)$ is purely nonatomic $[10$, p. 174]. Let $\delta=\bigcup_{\varepsilon \in \mathbf{P}} \delta_{\varepsilon}$. Then $\left\|A_{\mathbf{P}} \chi_{\delta}-\chi_{\delta}\right\|_{1}=\| \sum_{\varepsilon \in \mathbf{P}}\left(\int \chi_{\delta_{\varepsilon}} d v / v(\varepsilon) \chi_{\varepsilon}-\chi_{\delta}\left\|_{1}=\right\|(1 / 2)-\chi_{\delta} \|_{1}\right.$ $=1 / 2$, which is a contradiction. The converse is easily verified $\left({ }^{3}\right)$.

Proof of 4.1. Let $x \in E$ and $x^{\prime} \in \Omega(x)^{\prime}$ with $\left\langle x, x^{\prime}\right\rangle=1$. The mapping defined in 4.3 is easily seen to carry the interval $[-x, x]$ in $\mathfrak{M}_{R}(x)$ onto the interval $\{f \mid-1 \leqq f \leqq 1\}$ in $L^{1}\left(\left\langle\mu^{\sim}(\cdot) x, x^{\prime}\right\rangle\right)$, so this interval must be compact in $L^{1}\left(\left\langle\mu^{\sim}(\cdot) x, x^{\prime}\right\rangle\right)$ since $[-x, x]$ is closed and bounded in $E$. Thus the measure space $\left(X, \mathfrak{B},\left\langle\mu \sim(\cdot) x, x^{\prime}\right\rangle\right)$ satisfies the hypotheses of 4.4 , so $\left\langle\mu \sim(\cdot) x, x^{\prime}\right\rangle$ has atoms. By 4.2 it has point atoms, and thus so does $\mu^{\sim}$. Let $e$ be the supremum (in the sense of 3.17) of the projections corresponding to point atoms (i.e. the values of $\mu^{\sim}$ on those point atoms). If $e \neq 1$, then since $(1-e)$ commutes with $\mu^{\sim}$, the restriction of $\mu^{\sim}$ to the range of $(1-e)$ is a Borel spectral measure; since the closed bounded subsets of $(1-e) E$ are again compact, the restriction of $\mu \sim$ again possesses point atoms. Thus $\mu^{\sim}$ possesses point atoms $\xi$ for which $\mu^{\sim}(\{\xi\}) \cdot(1-e)$ differs from zero, which is absurd since $e$ dominates all point atoms of $\mu^{\sim}$.

COROLlary 4.5. Any Baire spectral measure triple on a Montel space E has the property of 4.1. For a Montel space is tonnelé and hence any spectral measure on it is equicontinuous, and bounded closed sets are compact.

COROLlaRY 4.6. If $E$ is a Montel space, then every equicontinuous $\sigma$-complete Boolean algebra on $E$ has a purely atomic completion equal to its strong closure in $\mathscr{L}(E)$.

Proof sketch. Such a Boolean algebra $\mathfrak{A}$ may be realized as the values of an equicontinuous Baire spectral measure triple $\left(X, \mathfrak{B}_{0}, \mu\right)$, where $X$ is the Stone space of $\mathfrak{A}$. The Borel extension $\left(X, \mathfrak{B}, \mu^{\sim}\right)$ of this triple has point atoms and takes values in the strong closure of $\mathfrak{A}$; clearly the family of all suprema of values of $\mu^{\sim}$ (suprema in the sense of 3.17) is a complete Boolean algebra contained in the strong closure of $\mathfrak{A}$.

For any idempotent $e$ in the strong closure of $\mathfrak{A}$ and any point atom $\xi \in X$ of $\mu^{\sim}$ we claim that $e \cdot \mu^{\sim}(\{\xi\})$ must be either $\mu^{\sim}(\{\xi\})$ or 0 ; for $e=\lim _{\alpha} \mu\left(\varepsilon_{\alpha}\right)$ for some net $\left\{\varepsilon_{\alpha}\right\}_{\alpha \in A}$ of clopen sets of $X$, and for each $\alpha \in A, \mu\left(\varepsilon_{\alpha}\right) \mu \sim(\{\xi\})=\mu \sim(\{\xi\})$ or 0 depending on whether $\xi \in \varepsilon_{\alpha}$ or not. If $P$ denotes the set of point atoms of $\underline{\mu \sim \text { in } X, \text { since }}$

(3) This elegant arrangement of the proof was suggested by G. L. Seever. 


$$
\begin{aligned}
1=\bigvee\left\{\mu^{\sim}(\{\xi\}) \mid \xi \in P\right\} \text { we have } e & =\bigvee\left\{e \cdot \mu^{\sim}(\{\xi\}) \mid \xi \in P\right\} \\
& =\bigvee\left\{\mu^{\sim}(\{\xi\}) \mid \xi \in P, e \cdot \mu^{\sim}(\{\xi\}) \neq 0\right\} .
\end{aligned}
$$

COROLlary 4.7. If $E$ is a Fréchet Montel space, then every o-complete Boolean algebra on $E$ is complete, equicontinuous, and purely atomic, and the set of atoms is countable.

Proof. Such a Boolean algebra $\mathfrak{A}$ is equicontinuous by 1.2 , and such a space is separable $\left[12\right.$, p. 373]; let $\left\{x_{n}\right\}_{n \in N}$ be dense in $E$. Since the restriction of $\mathfrak{U}$ to each $\mathfrak{M}\left(x_{n}\right)$ is countably decomposable, $\mathfrak{A}$ is countably decomposable and thus complete. So it equals its completion as constructed in 4.6 above, and by countable decomposability the set of atoms is countable.

This corollary is applicable to such spaces as the $C^{\infty}$ functions on an interval $[a, b]$, the holomorphic functions on a domain in $C$, etc.

COROLlaRY 4.8. Let $E$ be a locally convex space whose bounded closed subsets are compact, $\left(X, \mathfrak{B}_{0}, \mu\right)$ a Baire spectral measure triple in $\mathscr{L}(E)$ which is equicontinuous, $\left(X, \mathfrak{B}, \mu^{\sim}\right)$ its regular Borel extension. Then for any $\mathfrak{B}-$ measurable $f$ on $X$ and every $y$ in the domain of the (possibly unbounded) spectral operator $\int f d \mu^{\sim}\left(=\int f d \mu\right.$ if $f$ is Baire) we have

$$
\int f d \mu^{\sim}(y)=\sum_{\xi \in \mathbb{P}} f(\xi) \mu^{\sim}(\{\xi\}) y
$$

where the sum is meant in the sense of the limit of the net of finite sums. In particular, if $v$ is the measure on $C^{*}$ for which $\int f d \mu^{\sim}=\int z d v$, then

$$
\int f d \mu^{\sim}(y)=\sum_{\lambda \in \pi} \lambda v(\{\lambda\}) y
$$

where $\pi=\pi\left(\int f d \mu^{\sim}\right)$, the point spectrum of the operator.

Proof. For each finite subset $R \subseteq P$ it is easy to see that

$$
\left[\sum_{\xi \in R} \mu \sim(\{\xi\})\right]\left(\int f d \mu \sim(y)\right)=\sum_{\xi \in R} f(\xi) \mu \sim(\{\xi\}) y
$$

and the corollary follows by taking limits on both sides. It is known that the set of point atoms of $v$ is $\pi\left(\int f d \mu^{\sim}\right)$.

This proof can be modified to characterize the second commutator of a $\sigma$-complete equicontinuous Boolean algebra of idempotents. This modification will also yield another proof of 4.6.

We can also characterize the cyclic subspaces of $\sigma$-complete Boolean algebras on Fréchet nuclear spaces, as follows: 
Proposition 4.9. Let $E$ be a Fréchet nuclear space, $\mathfrak{A}$ a $\sigma$-complete Boolean algebra in $\mathscr{L}(E)$. Then each $\mathfrak{M}(x)$ is isomorphic to a "gestuften Raum" of Köthe $[12, p .408 f f$.$] in such a way that the formation of real and imaginary$ parts, absolute value and lattice order $\left(\right.$ on $\left.\mathfrak{M}_{R}(x)\right)$ correspond to the corresponding operations on the Köthe sequence space; the dual of $\mathfrak{M}(x)$ is thus isomorphic to the corresponding Stufenraum.

LEMMA 4.10. Let $E$ be a (real) nuclear space and locally convex vector lattice in the topology $\mathfrak{I}$. Then $\mathfrak{I}$ is generated by the seminorms $x \rightarrow\left\langle|x|, x^{\prime}\right\rangle$ for $x^{\prime}$ in the positive cone of $E^{\prime}$.

Proof. Clearly (by continuity of the absolute value function) the topology generated by these seminorms is no finer than $\mathfrak{I}$. On the other hand, by 1.6 $\mathfrak{I}$ is generated by seminorms of the form $x \rightarrow \sum_{i \in N} \lambda_{i}\left|\left\langle x, x_{i}{ }^{\prime}\right\rangle\right|$ where the $\lambda_{i}$ 's are positive and summable, and $\left\{x_{i}{ }^{\prime}\right\}_{i \in N}$ equicontinuous; but

$$
\sum_{i \in N} \lambda_{i}\left|\left\langle x, x_{i}{ }^{\prime}\right\rangle\right| \leqq\left\langle|x|, \sum_{i \in N} \lambda_{i}\left|x_{i}{ }^{\prime}\right|\right\rangle
$$

Proof of 4.9. Represent $\mathfrak{A}$ as the values of a Borel spectral measure triple $(X, \mathfrak{B}, \mu)$; enumerate the point atoms of $\mu$ as $\left\{\xi_{n}\right\}_{n \in N}$ (the case of finitely many point atoms is clear). For each $y=\int f d \mu(x) \in \mathfrak{M}(x)$ (represented as in 3.9), we have $y=\int f d \mu(x)=\sum_{n \in N} f\left(\xi_{n}\right) \mu\left(\left\{\xi_{n}\right\}\right) x$. Let $\eta_{n}=f\left(\xi_{n}\right)$ for $n \in N$. The correspondence between $y \in \mathfrak{M}(x)$ and $\left\{\eta_{n}\right\}_{n \in N}$ is easily seen to be biunivocal, preserve the vector-space operations, real and imaginary parts and absolute values and the lattice operations for real sequences. Similarly, for each $x^{\prime} \in \mathfrak{M}(x)^{\prime}$ let $\zeta_{n}=\left\langle\mu\left(\left\{\xi_{n}\right\}\right) x, x^{\prime}\right\rangle$; then $\left\langle y, x^{\prime}\right\rangle=\Sigma_{n \in N} \eta_{n}\left\langle\mu\left(\left\{\xi_{n}\right\}\right) x, x^{\prime}\right\rangle=\Sigma_{n \in N} \eta_{n} \zeta_{n}$, the series converging unconditionally. Again it is easy to see that the correspondence between $E^{\prime}$ and the sequences determined in this way is biunivocal on $\mathfrak{M}(x)^{\prime}$, takes the vector-space operations on $\mathfrak{M}(x)^{\prime}$ into the coördinatewise operations on sequences, with the real-valued sequences identifiable with $\mathfrak{M}_{R}(x)^{\prime}$. Now by 4.10 (since $\mathfrak{M}_{R}(x)$ is metrizable) there exists a sequence $\left\{x_{m}{ }^{\prime}\right\}_{m \in N}$ of elements of $\boldsymbol{\Omega}(x)^{\prime}$ for which the seminorms $\left\{x \rightarrow\left\langle|x|, x_{m}{ }^{\prime}\right\rangle\right\}_{m \in N}$ generate the topology of $\mathfrak{M}_{R}(x)$, and there is no loss of generality in assuming $x_{m}{ }^{\prime} \leqq x_{m+1}{ }^{\prime}$. It is also easy to see that these seminorms generate the topology of $\mathfrak{M}(x)$ if the absolute value function used is that on $\mathfrak{M}(x)$ instead of the lattice absolute value function on $\mathfrak{M}_{R}(x)$. The sequences $\left\{\zeta_{m, n}\right\}_{n \in N}$ corresponding to the $\left\{x_{m}{ }^{\prime}\right\}$ increase coördinatewise with $m$, and $\left\langle|y|, x_{m}{ }^{\prime}\right\rangle=\sum_{n \in N}\left|\eta_{n}\right| \zeta_{m, n}$. In particular, $\sum_{n \in N} \eta_{n} \mu\left(\left\{\xi_{n}\right\}\right) x$ converges in $E$ if and only if the scalar series $\Sigma_{n \in N}\left|\eta_{n}\right| \zeta_{m, n}$ converge for all $m \in N$, and the dual space of $\mathfrak{M}(x)$ can be identified with the space of all sequences $\left\{\theta_{n}\right\}_{n \in N}$ of scalars for which there exist an $m$ and a constant $k$ with $\left|\theta_{n}\right| \leqq k \cdot \zeta_{m, n}$ for all $n \in N$. Thus $\mathfrak{M}(x)$ and $\mathfrak{M}(x)^{\prime}$ are represented respectively as the gestufter Raum and Stufenraum associated with the Stufen 
$\left\{\zeta_{m, n}\right\}_{n \in N}$, as in [12, p. $422 \mathrm{ff}$.]. The topology of $\mathfrak{M}(x)$ corresponds to the "normal topology" of the gestuften Raum.

In conclusion we give the following elementary characterization of the spectral measures on the spaces $\omega_{d}$ and $\phi_{d}$, product and locally convex direct sum respectively of $d$ copies of $C$. It suffices to characterize those on $\phi_{d}$, since the two spaces are each other's topological duals and both are reflexive; cf. the duality results of [17]. Since the topology of $\phi_{d}$ is its finest locally convex topology, its bounded subsets are finite-dimensional. Let $(X, \mathfrak{B}, \mu)$ be a regular Borel spectral measure triple in $\mathscr{L}\left(\phi_{d}\right) ; \mu$ is equicontinuous since $\phi_{d}$ is tonnelé. Since $\phi_{d}$ is Montel, $\mu$ has point atoms; let $P$ be the set of all point atoms of $\mu$ in $X$. Then for each $x \in \phi_{d}$ the set $\{\mu(\{\xi\}) x \mid \xi \in P\}$ lies in a finite-dimensional subspace, since it is bounded; but since its elements are linearly independent, at most finitely many of them are nonzero, and $x$ is their sum. Thus $\bigcup_{\xi \in P} \mu(\{\xi\})\left[\phi_{d}\right]$ generates $\phi_{d}$, algebraically, and so contains a Hamel basis of $\phi_{d}$; the cardinality of Hamel bases being determined by the space, one may assume the basis indexed by $d$, say $\left\{x_{\eta}\right\}_{\eta \in d}$. For each $\eta \in d$ there is a unique $\xi(\eta) \in P$ for which $x_{\eta} \in \mu(\{\xi(\eta)\})\left[\phi_{d}\right]$. Let $v$ be the isomorphism of $\phi_{d}$ with itself which sends the $\eta$ th element of the standard basis of $\phi_{d}$ to $x_{\eta}$; then for each $\delta \in \mathfrak{B}$ the idempotent $v^{-1} \mu(\delta) v$ is the projection of $\phi_{d}$ which sends the $\eta$ th element of the standard basis of $\phi_{d}$ to itself or zero according to whether $\xi(\eta) \in \delta$ or not. So the spectral measure $v^{-1} \mu(\cdot) v$ takes its values in the set of projections onto "coördinate subspaces" of $\phi_{d}$, i.e. subspaces determined by subsets $s \subseteq d$ in the obvious way. Now if $(X, \mathfrak{B}, \mu)$ is a regular Borel measure in $\mathscr{L}\left(\omega_{d}\right)$, then $\mu(\cdot)^{\prime}$ is a regular Borel spectral measure in $\mathscr{L}\left(\phi_{d}\right)$ to which the preceding considerations apply, so there exists a topological automorphism $v^{\prime} \in \mathscr{L}\left(\omega_{d}\right)$ for which the spectral measure $v^{\prime} \mu(\cdot) v^{\prime-1}$ takes its values in the set of projections on coördinate subspaces of $\omega_{d}$, by duality. And it is easy to verify that for each spectral operator $\int f d \mu$ associated with $\mu$, the corresponding operator $v^{\prime}\left[\int f d \mu\right] v^{\prime-1}$ has a diagonal matrix with respect to the coordinates of $\omega_{d}$, and similarly for $\phi_{d}$. Summarizing, then,

PROPOSITION 4.11. If $(X, \mathfrak{B}, \mu)$ is a regular Borel spectral measure triple in $\mathscr{L}\left(\omega_{d}\right)$ or $\mathscr{L}\left(\phi_{d}\right)$, then there exists a topological automorphism $v$ on $\omega_{d}$ or $\phi_{d}$ respectively, such that the values of $v \cdot \mu(\cdot) \cdot v^{-1}$ are projections on coördinate subspaces of $\omega_{d}$ or $\phi_{d}$ respectively. Every spectral operator associated with $(X, \mathfrak{B}, \mu)$ is thus similar to an operator with diagonal matrix.

COROLlaRY 4.12. If $\mathfrak{A}$ is a complete equicontinuous Boolean algebra of projections on $\omega_{d}$ or $\phi_{d}$ respectively, then there exists a topological automorphism $v$ of $\omega_{d}$ or $\phi_{d}$ for which $v \mathfrak{A} v^{-1}=\left\{v e v^{-1} \mid e \in \mathfrak{A}\right\}$ is a complete Boolean algebra of projections on coördinate subspaces. If $d=\aleph_{0}, \sigma$-completeness suffices instead of completeness and equicontinuity. 


\section{REFERENCES}

1. W. Bade, Unbounded spectral operators, Pacific J. Math. 4 (1954), 373-392.

2. - Weak and strong limits of spectral operators, Pacific J. Math. 4 (1954), 393-413.

3. - On Boolean algebras of projections and algebras of operators, Trans. Amer. Math. Soc. 80 (1955), 345-360.

4. - - A multiplicity theory for Boolean algebras of projections in Banach spaces, Trans. Amer. Math. Soc. 92 (1959), 508-530.

5. R. Bartle, N. Dunford and J. Schwartz, Weak compactness and iector measures, Canad. J. Math. 7 (1955), 289-305.

6. N. Bourbaki, Espaces vectoriels topologiques, Chapters I-II and III-V, Actualités Sci. Indust. No. 1189 and No. 1229, Hermann, Paris, 1953, 1955.

7. N. Dunford, Spectral operators, Pacific J. Math. 4 (1954), 321-354.

8. N. Dunford and J. Schwartz, Linear operators. I, General Theory, Interscience, New York, 1958.

9. A. Grothendieck, Produits tensoriels topologiques et espaces nucléaires, Mem. Amer. Math. Soc. No. 16 (1955), 140 pp.

10. P. Halmos, Measure theory, Van Nostrand, New York, 1950.

11. S. Kakutani, An example concerning uniform boundedness of spectral measures, Pacific J. Math. 4 (1954), 363-372.

12. G. Köthe, Topologische lineare Räume. I, Springer, Berlin, 1960.

13. F. Riesz and B. Sz.-Nagy, Functional analysis, Ungar, New York, 1955.

14. H. Schaefer, Halbgeordnete lokalkonvexe Vektorräume, Math. Ann. 135 (1958), 115-141.

15. — Halbgeordnete lokalkonvexe Vektorräume. II, Math. Ann. 138 (1959), 259-286.

16. - - Halbgeordnete lokalkonvexe Vektorräume. III, Math. Ann. 141 (1960), 113-142.

17. - Spectral measures in locally convex algebras, Acta Math. 107 (1962), 125-173.

18. H. Schaefer and B. Walsh, Spectral operators in spaces of distributions, Bull. Amer. Math. Soc. 68 (1962), 509-511.

19. J. Schwartz, Perturbations of spectral operators and applications. I, Pacific J. Math. 4 (1954), 415-458.

20. J. Wermer, Commuting spectral measures on Hilbert space, Pacific J. Math. 4 (1954), $355-361$.

UNIVERSITY OF CALIFORNIA,

Los Angeles, California 